\title{
ON $d$-ORTHOGONAL TCHEBYCHEV POLYNOMIALS, II
}

\author{
Khalfa Douak and Pascal Maroni
}

\begin{abstract}
In Part I, we considered the following problem: Find all $d$-orthogonal polynomial sequences $\left\{P_{n}\right\}_{n \geq 0}$ such that $P_{n}^{(1)}=P_{n}, n=0,1,2, \ldots$, where $\left\{P_{n}^{(1)}\right\}_{n \geq 0}$ is the associated polynomial sequence of $\left\{P_{n}\right\}_{n \geq 0}$. The resulting polynomials are an extension of the classical Tchebychev polynomials of the second kind. A detailed study was made in the particular case $d=2$.

The purpose of this paper is to make similar investigations by considering the analogous problem: Find all $d$-orthogonal polynomial sequences $\left\{P_{n}\right\}_{n \geq 0}$ such that $P_{n}^{(1)}=Q_{n}, n=0,1,2, \ldots$, where $Q_{n}(x):=(n+1)^{-1} P_{n+1}^{\prime}(x), n \geq$ 0 . Here, we show that the resulting polynomials are a natural extension of the classical Tchebychev polynomials of the first kind. The recurrence coefficients and generating function are determined explicitly. As in Part I, a detailed study will be carried out for the case $d=2$. A third-order differential equation and a differential-recurrence relation are given. Again, in the particular case, integral representations are obtained of the linear functionals with respect to which the resulting polynomials are 2 -orthogonal.
\end{abstract}

\section{Introduction and preliminary results}

This work represents a continuation of our previous paper [6]. The terminology and notations employed here are the same as those in the Part I.

It is well known (see e.g., [2]) that the Tchebychev monic polynomials $\left\{\widehat{T}_{n}\right\}_{n \geq 0}$ and $\left\{\widehat{U}_{n}\right\}_{n \geq 0}$ of the first and second kinds, respectively, are linked, at the same time, by the two relations

$$
\widehat{T}_{n}^{(1)}(x)=\widehat{U}_{n}(x) \text { and } \widehat{T}_{n+1}^{\prime}(x)=(n+1) \widehat{U}_{n}(x), n=0,1, \ldots,
$$

where $\left\{\widehat{T}_{n}^{(1)}\right\}_{n \geq 0}$ are the associated polynomials of $\left\{\widehat{T}_{n}\right\}_{n \geq 0}$. The polynomials $\left\{\widehat{T}_{n}\right\}_{n \geq 0}$ are orthogonal with respect to the weight function $1 / \sqrt{1-x^{2}}$ on $[-1,+1]$, that is,

$$
\frac{1}{\pi} \int_{-1}^{1} \widehat{T}_{n}(x) \widehat{T}_{m}(x) \frac{1}{\sqrt{1-x^{2}}} d x=k_{n} \delta_{n, m}, \quad k_{n}=2^{1-2 n-\delta_{n, 0}}, \quad n, m \geq 0,
$$

and they satisfy the second-order recurrence relation

$$
\begin{aligned}
\widehat{T}_{n+2}(x) & =x \widehat{T}_{n+1}(x)-\frac{2^{\delta_{n, 0}}}{4} \widehat{T}_{n}(x), \quad n \geq 0 \\
\widehat{T}_{0}(x) & =1, \quad \widehat{T}_{1}(x)=x
\end{aligned}
$$

Received October 16, 1996, revised May 14, 1997.

1991 Mathematics Subject Classification: 42C05, 33C45.

Key words and phrases: Orthogonal polynomials, $d$-orthogonality, Tchebychev polynomials, recurrence relations, hypergeometric functions, integral representations. 
The associated polynomials $\widehat{T}_{n}^{(1)}, n=0,1,2, \ldots$, are defined by

$$
\widehat{T}_{n}^{(1)}(x)=\frac{1}{\pi} \int_{-1}^{1} \frac{\widehat{T}_{n+1}(x)-\widehat{T}_{n+1}(\xi)}{x-\xi} \frac{1}{\sqrt{1-\xi^{2}}} d \xi, \quad n=0,1,2, \ldots,
$$

and satisfy the second-order recurrence relation

$$
\begin{aligned}
& \widehat{T}_{n+2}^{(1)}(x)=x \widehat{T}_{n+1}^{(1)}(x)-\frac{1}{4} \widehat{T}_{n}^{(1)}(x), \quad n \geq 0, \\
& \widehat{T}_{0}^{(1)}(x)=1, \quad \widehat{T}_{1}^{(1)}(x)=x .
\end{aligned}
$$

The connection between the two kinds of the classical Tchebychev polynomials was the first motivation for us to consider problems of the types

$$
P_{n}^{(1)}=P_{n}, \quad n=0,1,2, \ldots
$$

or

$$
P_{n}^{(1)}=Q_{n}, \quad n=0,1,2, \ldots
$$

and to look for the resulting polynomials in the $d$-orthogonality case. These polynomials are natural extensions of the classical Tchebychev polynomials. They have some properties that are analogous to those of the classical Tchebychev polynomials. In the case $d=2$, for example, they are related to weight functions involving the square root of a polynomial of degree 3, plus Stieltjes-type functions suitably chosen, whereas Tchebychev polynomials are related to weight functions involving the square root of a polynomial of degree 2 .

In this work, we investigate the sequences of monic polynomials $\left\{P_{n}\right\}_{n \geq 0}$ for which (1.2) holds. The organization of the paper is as follows. Section 2 is devoted to the statement and the solution of the problem (1.2) in the two special cases $d=1$ and $d=2$. For $d=1$, we obtain that $\left\{P_{n}\right\}_{n \geq 0}$ are the Tchebychev monic polynomials of the first kind, and for $d=2$, we obtain the analogous 2-orthogonal polynomials. Among such polynomials, the 2-symmetric ones (see Definition 2.1 below) are singled out and called 2-orthogonal Tchebychev polynomials of the first kind. In Section 3, for $d=2$, we show that the resulting polynomials satisfy a differential-recurrence relation, and in the 2-symmetric case, we obtain that each polynomial satisfies a third-order differential equation. Finally, Section 4 deals with the problem of integral representations of the linear functionals associated with the 2-orthogonal polynomials obtained in Section 2, as well as, the functionals involved in the cubic decomposition of such polynomials.

Let $\left\{P_{n}\right\}_{n \geq 0}$ be a sequence of monic polynomials, $\left\{u_{n}\right\}_{n \geq 0}$, the dual sequence associated with $\left\{P_{n}\right\}_{n \geq 0}$ defined by $\left\langle u_{n}, P_{n}\right\rangle=\delta_{n, m}, n, m \geq 0$, where $\langle$,$\rangle is the$ duality bracket between the vector space of polynomials with complex coefficients and its dual (see Subsection 1.1. below).

Lemma 1.1. [12] For any linear functional $u$ and integer $p \geq 1$, the following two statements are equivalent.

(a) $\left\langle u, P_{p-1}\right\rangle \neq 0 ;\left\langle u, P_{n}\right\rangle=0$ for $n \geq p ;$

(b) there exists $\lambda_{\nu} \in \mathbb{C}, 0 \leq \nu \leq p-1, \lambda_{p-1} \neq 0$, such that $u=\sum_{\nu=0}^{p-1} \lambda_{\nu} u_{\nu}$. 
Definition 1.1. Let $\omega_{1}, \omega_{2}, \ldots, \omega_{d}$ be $d$ linear functionals $(d \geq 1)$. The polynomial sequence $\left\{P_{n}\right\}_{n \geq 0}$ is called a $d$-orthogonal polynomial sequence $(d-$ OPS $)$ with respect to $\Omega={ }^{t}\left(\omega_{1}, \ldots, \omega_{d}\right)$ if it fulfills $[11,13]$

$$
\begin{aligned}
\left\langle\omega_{\alpha}, P_{m} P_{n}\right\rangle & =0, \quad n \geq m d+\alpha, \\
\left\langle\omega_{\alpha}, P_{m} P_{m d+\alpha-1}\right\rangle & \neq 0,
\end{aligned}
$$

for each integer $\alpha$ with $\alpha=1,2, \ldots, d$ and $m \geq 0$.

Remark 1.1. (a) When $d=1$, we again have ordinary regular orthogonality. In this case $\left\{P_{n}\right\}_{n \geq 0}$ is an orthogonal polynomial sequence (OPS).

(b) The inequalities (1.4) are the regularity conditions (for equivalent conditions of regularity, see, e.g., [11, p.110]). In this case, the $d$-dimensional functional $\Omega$ is called regular. It is not unique. Indeed, according to Lemma 1.1, we have

$$
\begin{array}{rlrl}
\omega_{\alpha} & =\sum_{\nu=0}^{\alpha-1} \lambda_{\nu}^{\alpha} u_{\nu}, & \lambda_{\alpha-1}^{\alpha} \neq 0, \quad 1 \leq \alpha \leq d \\
\Longleftrightarrow u_{\nu} & =\sum_{\alpha=1}^{\nu+1} \tau_{\alpha}^{\nu} \omega_{\alpha}, \quad \tau_{\nu+1}^{\nu} \neq 0, \quad 0 \leq \nu \leq d-1 .
\end{array}
$$

Therefore, from now on, we shall work uniquely with the dual functionals $u_{0}, \ldots, u_{d-1}$, that is to say, $\mathcal{U}={ }^{t}\left(u_{0}, \ldots, u_{d-1}\right)$; it is the canonical regular $d$-dimensional functional with respect to which $\left\{P_{n}\right\}_{n \geq 0}$ is $d$-orthogonal.

A remarkable characterization of the $d$-OPS is that they satisfy a $(d+1)$-order recurrence relation [13], which we write in the form

$$
P_{m+d+1}(x)=\left(x-\beta_{m+d}\right) P_{m+d}(x)-\sum_{\nu=0}^{d-1} \gamma_{m+d-\nu}^{d-1-\nu} P_{m+d-1-\nu}(x), \quad m \geq 0
$$

with the initial conditions

$$
\begin{aligned}
& P_{0}(x)=1, \quad P_{1}(x)=x-\beta_{0}, \quad \text { and if } d \geq 2, \\
& P_{n}(x)=\left(x-\beta_{n-1}\right) P_{n-1}(x)-\sum_{\nu=0}^{n-2} \gamma_{n-1-\nu}^{d-1-\nu} P_{n-2-\nu}(x), \quad 2 \leq n \leq d,
\end{aligned}
$$

and the regularity conditions

$$
\gamma_{m+1}^{0} \neq 0, \quad m \geq 0 .
$$

The above recurrence relation is equivalent to the following recurrence relation between $d+2$ consecutive linear functionals [12]:

$$
x u_{n}=u_{n-1}+\beta_{n} u_{n}+\sum_{\nu=0}^{d-1} \gamma_{n+1}^{d-1-\nu} u_{n+1+\nu}, \quad n \geq 0 \quad\left(u_{-1}=0\right) .
$$

Another characterization has been given in [11].

Note that when $d=1$, the recurrence (1.5) with (1.6) is reducible to the secondorder recurrence relation

$$
\begin{aligned}
P_{n+2}(x) & =\left(x-\beta_{n+1}\right) P_{n+1}(x)-\gamma_{n+1} P_{n}(x), \quad n \geq 0, \\
P_{0}(x) & =1, \quad P_{1}(x)=x-\beta_{0} .
\end{aligned}
$$


Now, the polynomial sequence $\left\{P_{n}\right\}_{n \geq 0}$ is called the "classical" $d$-OPS, or simply $d$-classical, if it satisfies the Hahn property, that is to say, the sequence $\left\{Q_{n}\right\}_{n \geq 0}$ is also $d$-orthogonal where $Q_{n}(x):=(n+1)^{-1} P_{n+1}^{\prime}(x)$ is the monic derivative. In this case $\left\{Q_{n}\right\}_{n \geq 0}$ also satisfies a $(d+1)$-order recurrence relation, namely

$$
Q_{m+d+1}(x)=\left(x-\widetilde{\beta}_{m+d}\right) Q_{m+d}(x)-\sum_{\nu=0}^{d-1} \widetilde{\gamma}_{m+d-\nu}^{d-1-\nu} Q_{m+d-1-\nu}(x), \quad m \geq 0,
$$

with the initial and the regularity conditions

$$
\begin{aligned}
& Q_{0}(x)=1, \quad Q_{1}(x)=x-\widetilde{\beta}_{0} \quad \text { and if } d \geq 2, \\
& Q_{n}(x)=\left(x-\widetilde{\beta}_{n-1}\right) Q_{n-1}(x)-\sum_{\nu=0}^{n-2} \widetilde{\gamma}_{n-1-\nu}^{d-1-\nu} Q_{n-2-\nu}(x), 2 \leq n \leq d, \\
& \widetilde{\gamma}_{m+1}^{0} \neq 0, \quad m \geq 0,
\end{aligned}
$$

and it is $d$-orthogonal with respect to $\mathcal{V}={ }^{t}\left(v_{0}, \ldots, v_{d-1}\right)$, where $\left\{v_{n}\right\}_{n \geq 0}$ is the dual sequence of $\left\{Q_{n}\right\}_{n \geq 0}$.

1.1. Some elementary operations in $\mathcal{P}$ and $\mathcal{P}^{\prime}$. Let $\mathcal{P}$ be the vector space of polynomials with coefficients in $\mathbb{C}$ and let $\mathcal{P}^{\prime}$ be its dual. We denote by $\langle u, f\rangle$ the effect of $u \in \mathcal{P}^{\prime}$ on $f \in \mathcal{P}$. In particular, we denote the moments of $u$ by $(u)_{n}=\left\langle u, x^{n}\right\rangle$, $n \geq 0$.

By transposition, we obtain

(a) The left-multiplication of a functional by a polynomial:

$$
\langle f u, p\rangle:=\langle u, f p\rangle, \quad u \in \mathcal{P}^{\prime}, \quad f, p \in \mathcal{P} .
$$

(b) The derivative of a functional:

$$
\langle D u, p\rangle=\left\langle u^{\prime}, p\right\rangle:=-\left\langle u, p^{\prime}\right\rangle, \quad u \in \mathcal{P}^{\prime}, \quad p \in \mathcal{P} .
$$

An APPLiCATION. We have [11]

$$
D v_{n}=-(n+1) u_{n+1}, \quad n \geq 0,
$$

where $\left\{u_{n}\right\}_{n \geq 0}$ (resp., $\left\{v_{n}\right\}_{n \geq 0}$ ) is the dual sequence of $\left\{P_{n}\right\}_{n \geq 0}$ (resp., $\left\{Q_{n}\right\}_{n \geq 0}$ ).

(c) The division of a functional by a polynomial:

From the linear application $p \rightarrow\left(\theta_{c} p\right)(x)=\frac{p(x)-p(c)}{x-c}, c \in \mathbb{C}$, we have

$$
\left\langle(x-c)^{-1} u, p\right\rangle:=\left\langle u, \theta_{c} p\right\rangle \text {. }
$$

(d) The right-multiplication of a functional by a polynomial:

$$
(u, p) \rightarrow(u p)(x)=\left\langle u, \frac{x p(x)-\xi p(\xi)}{x-\xi}\right\rangle=\sum_{\nu=0}^{n}\left(\sum_{\mu=\nu}^{n} a_{\mu}(u)_{\mu-\nu}\right) x^{\nu}, u \in \mathcal{P}^{\prime}
$$

and

$$
p(x)=\sum_{\nu=0}^{n} a_{\nu} x^{\nu}
$$

(e) The product of two functionals:

$$
\langle u v, p\rangle:=\langle u, v p\rangle, u, v \in \mathcal{P}^{\prime}, p \in \mathcal{P} .
$$


(f) The operator $\sigma_{s}$ :

Let us introduce the operator $\sigma_{s}: \mathcal{P} \rightarrow \mathcal{P} ; s \in \mathbb{N}$, defined by

$$
\left(\sigma_{s} f\right)(x):=f\left(x^{s}\right), \quad f \in \mathcal{P} \text {. }
$$

By transposition, we define ${ }^{t} \sigma_{s}$ by

$$
\left\langle{ }^{t} \sigma_{s}(u), f\right\rangle=\left\langle u, \sigma_{s} f\right\rangle, \quad u \in \mathcal{P}^{\prime}, \quad f \in \mathcal{P},
$$

so that $\left({ }^{t} \sigma_{s}(u)\right)_{n}=(u)_{s n}, n \geq 0$. Therefore, from now on, we set ${ }^{t} \sigma_{s}=\sigma_{s}$, then $\left\langle\sigma_{s}(u), f\right\rangle=\left\langle u, \sigma_{s} f\right\rangle, f \in \mathcal{P} ; u \in \mathcal{P}^{\prime}$, so that $\left(\sigma_{s}(u)\right)_{n}=(u)_{s n}, n \geq 0$.

Lemma 1.2. [6] For all $f \in \mathcal{P}$ and $u \in \mathcal{P}^{\prime}$, we have the properties:

$$
\begin{aligned}
f(x) \sigma_{s}(u) & =\sigma_{s}\left(f\left(x^{s}\right) u\right), \\
s D\left(\sigma_{s}(u)\right) & =\sigma_{s}\left(D\left(x^{1-s} u\right)\right), \\
\sigma_{s}(D u) & =s D\left(\sigma_{s}\left(x^{s-1} u\right)\right) .
\end{aligned}
$$

\subsection{The associated polynomials.}

Definition 1.2. We call the associated sequence of $\left\{P_{n}\right\}_{n \geq 0}$ (with respect to $u_{0}$ ), the sequence $\left\{P_{n}^{(1)}\right\}_{n \geq 0}$ defined by

$$
P_{n}^{(1)}(x)=\left\langle u_{0}, \frac{P_{n+1}(x)-P_{n+1}(\xi)}{x-\xi}\right\rangle, \quad n \geq 0 .
$$

Every polynomial $P_{n}^{(1)}$ is monic. We denote by $\left\{u_{n}^{(1)}\right\}_{n \geq 0}$, the dual sequence of $\left\{P_{n}^{(1)}\right\}_{n \geq 0}$.

Proposition 1.1. [12] We have

$$
u_{n}^{(1)}=\left(x u_{n+1}\right) u_{0}^{-1}, \quad n \geq 0,
$$

where $u^{-1}$ (the inverse element of $u$ ) exists if and only if $(u)_{0} \neq 0$, and then $u u^{-1}=\delta$.

Now, the successive associated sequences are defined by recurrence $P_{n}^{(r+1)}=\left(P_{n}^{(r)}\right)^{(1)}$ and $u_{n}^{(r+1)}=\left(u_{n}^{(r)}\right)^{(1)}$, for $n, r \geq 0$.

Remark 1.2. When $\left\{P_{n}\right\}_{n \geq 0}$ is an OPS (with respect to $u_{0}$ ), then it satisfies the recurrence relation (1.9), and we know that the associated sequence $\left\{P_{n}^{(1)}\right\}_{n \geq 0}$ satisfies the recurrence relation

$$
\begin{aligned}
P_{n+2}^{(1)}(x) & =\left(x-\beta_{n+2}\right) P_{n+1}^{(1)}(x)-\gamma_{n+2} P_{n}^{(1)}(x), n \geq 0, \\
P_{0}^{(1)}(x) & =1, P_{1}^{(1)}(x)=x-\beta_{1},
\end{aligned}
$$

and it is orthogonal with respect to $u_{0}^{(1)}$. It is clear that $P_{n}^{(0)}=P_{n}, n \geq 0$, and $u_{0}^{(0)}=u_{0}$.

Likewise, if $\left\{P_{n}\right\}_{n \geq 0}$ is $d$-OPS, namely, if it satisfies the recurrence (1.5)-(1.6) then $\left\{P_{n}^{(1)}\right\}_{n \geq 0}$ also satisfies the $(d+1)$-order recurrence relation

$$
P_{m+d+1}^{(1)}(x)=\left(x-\beta_{m+d+1}\right) P_{m+d}^{(1)}(x)-\sum_{\nu=0}^{d-1} \gamma_{m+d-\nu+1}^{d-1-\nu} P_{m+d-1-\nu}^{(1)}(x), \quad m \geq 0,
$$


with the initial conditions

$$
\begin{aligned}
& P_{0}^{(1)}(x)=1, \quad P_{1}^{(1)}(x)=x-\beta_{1}, \quad \text { and if } d \geq 2 \\
& P_{n}^{(1)}(x)=\left(x-\beta_{n}\right) P_{n-1}^{(1)}(x)-\sum_{\nu=0}^{n-2} \gamma_{n-\nu}^{d-1-\nu} P_{n-2-\nu}^{(1)}(x), \quad 2 \leq n \leq d .
\end{aligned}
$$

Thus, we have the evident result:

Proposition 1.2. When $\left\{P_{n}\right\}_{n \geq 0}$ is d-OPS with respect to $\mathcal{U}={ }^{t}\left(u_{0}, \ldots, u_{d-1}\right)$, then $\left\{P_{n}^{(1)}\right\}_{n \geq 0}, r \geq 1$, is d-OPS with respect to $\mathcal{U}^{(1)}={ }^{t}\left(u_{0}^{(1)}, \ldots, u_{d-1}^{(1)}\right)$.

\section{Statement of the problem}

We pose the analogous general problem as follows.

For fixed integer $r$, find all d-OPS which satisfy

$$
P_{n}^{(r)}(x)=Q_{n}(x), \quad n=0,1,2, \ldots
$$

Remark 2.1. The $d$-OPS $\left\{P_{n}\right\}_{n \geq 0}$ is necessarily "classical" (in the sense of the Hahn property, that is, $\left\{Q_{n}\right\}_{n \geq 0}$ is also $d$-OPS). Indeed, since $\left\{P_{n}\right\}_{n \geq 0}$ is $d$-OPS, $\left\{P_{n}^{(r)}\right\}_{n \geq 0}$ is also $d$-OPS, and then $\left\{Q_{n}\right\}_{n \geq 0}$ is a $d$-OPS. This general problem has remained unsolved. However, for some special choices of the integers $r$ and $d$, the resulting polynomials are known.

EXAMPLES. In the case $r=0$, the equality (2.1) becomes $P_{n}(x)=Q_{n}(x), n=$ $0,1,2, \ldots$, and $\left\{P_{n}\right\}_{n \geq 0}$ is an Appell polynomial sequence, so the above problem becomes

Find all polynomial sequences which are at the same time d-OPS and Appell polynomials.

(1) When $d=1$ (ordinary orthogonality), the only resulting polynomials are the Hermite polynomials (up to a shift) (see, e.g., [1]).

(2) In the $d$-orthogonality case, the problem is solved for $d=2$ by the authors in [4], and recently, the solution is given for any integer (dimension) $d(d \geq 1)$ by the first author in [3]. The resulting polynomials are a natural extension of the Hermite polynomials. Among such polynomials, the $d$-symmetric ones are singled out. They are named $d$-orthogonal Hermite polynomials.

Here and in the sequel, we investigate the case $r=1$, namely, we look for all $d$-OPS which satisfy

$$
P_{n}^{(1)}(x)=Q_{n}(x), \quad n \geq 0
$$

The method of proof is based only on the solution of the nonlinear system satisfied by the recurrence coefficients. For the sake of simplicity, we only consider the two special cases $d=1, d=2$. 


\subsection{Special cases.}

2.1.1. The case $d=1$. (ordinary orthogonality) In this case the problem becomes: Find all polynomial sets which are OPS and which satisfy (2.2).

First, since $\left\{P_{n}\right\}_{n \geq 0}$ is OPS, then both $\left\{P_{n}\right\}_{n \geq 0}$ and $\left\{Q_{n}\right\}_{n \geq 0}$ satisfy, respectively, the following second-order recurrence relations:

$$
\begin{aligned}
P_{n+2}(x) & =\left(x-\beta_{n+1}\right) P_{n+1}(x)-\gamma_{n+1} P_{n}(x), \quad\left(\gamma_{n+1} \neq 0\right), \quad n \geq 0, \\
P_{0}(x) & =1, \quad P_{1}(x)=x-\beta_{0},
\end{aligned}
$$

and

$$
\begin{aligned}
Q_{n+2}(x) & =\left(x-\widetilde{\beta}_{n+1}\right) Q_{n+1}(x)-\widetilde{\gamma}_{n+1} Q_{n}(x), \quad\left(\widetilde{\gamma}_{n+1} \neq 0\right), \quad n \geq 0, \\
Q_{0}(x) & =1, \quad Q_{1}(x)=x-\widetilde{\beta}_{0} .
\end{aligned}
$$

Then, by virtue of the recurrence relations (1.21) and (2.4), the sequence $\left\{P_{n}\right\}_{n \geq 0}$ fulfills (2.2) if and only if

$$
\begin{aligned}
\tilde{\beta}_{n} & =\beta_{n+1}, & & n \geq 0, \\
\widetilde{\gamma}_{n+1} & =\gamma_{n+2}, & & n \geq 0 .
\end{aligned}
$$

Next, from (2.3), (2.4) and after some manipulations, it is possible to show that the sequences $\left\{\beta_{n}\right\}_{n \geq 0},\left\{\widetilde{\beta}_{n}\right\}_{n \geq 0},\left\{\gamma_{n}\right\}_{n \geq 1}$, and $\left\{\widetilde{\gamma}_{n}\right\}_{n \geq 1}$ satisfy the system (see, e.g., [4])

$$
\begin{aligned}
(n+3) \widetilde{\beta}_{n+1} & =(n+1) \widetilde{\beta}_{n}+(n+2) \beta_{n+2}-n \beta_{n+1}, & & n \geq 0, \\
2 \widetilde{\beta}_{0} & =\beta_{0}+\beta_{1}, & & \\
(n+3) \widetilde{\gamma}_{n+1}-(n+1) \widetilde{\gamma}_{n} & =(n+1) \gamma_{n+2}-(n-1) \gamma_{n+1} & & \\
& +(n+1)\left(\beta_{n+1}-\widetilde{\beta}_{n}\right)^{2}, & & n \geq 1, \\
3 \widetilde{\gamma}_{1}= & \gamma_{2}+\gamma_{1}+\left(\beta_{1}-\widetilde{\beta}_{0}\right)^{2}, & & \\
n \gamma_{n+1}\left(\beta_{n+1}+\beta_{n}-2 \widetilde{\beta}_{n-1}\right) & =(n+1) \widetilde{\gamma}_{n}\left(2 \beta_{n+1}-\widetilde{\beta}_{n}-\widetilde{\beta}_{n-1}\right), & & n \geq 1, \\
(n+2) \widetilde{\gamma}_{n+1} \widetilde{\gamma}_{n}+n \gamma_{n+2} \gamma_{n+1} & =2(n+1) \gamma_{n+2} \widetilde{\gamma}_{n}, & & n \geq 1 .
\end{aligned}
$$

From this, by using (2.5), we easily obtain that

$$
P_{n}^{(1)}(x)=Q_{n}(x), \quad n \geq 0 \Longleftrightarrow \begin{cases}\beta_{n}=\beta_{0}, & n \geq 0, \\ \gamma_{n+1}=\frac{1}{2} \gamma_{1}, & n \geq 1 .\end{cases}
$$

We always can choose $\beta_{0}=0$ and $\gamma_{1}=1 / 2$; then the recurrence relation (2.3) (resp., (2.4)) becomes

$$
\begin{aligned}
P_{n+2}(x) & =x P_{n+1}(x)-\frac{2^{\delta_{n, 0}}}{4} P_{n}(x), \quad n \geq 0 \\
P_{0}(x) & =1, \quad P_{1}(x)=x
\end{aligned}
$$

respectively,

$$
\begin{aligned}
Q_{n+2}(x) & =x Q_{n+1}(x)-\frac{1}{4} Q_{n}(x), \quad n \geq 0, \\
Q_{0}(x) & =1, \quad Q_{1}(x)=x .
\end{aligned}
$$

Hence, $\left\{P_{n}\right\}_{n \geq 0}$ (resp., $\left\{Q_{n}\right\}_{n \geq 0}$ ) is the monic Tchebychev OPS of the first (resp., second) kind. 
2.1.2. The case $d=2$. (2-orthogonality)

Now, we state and solve the similar problem with $d=2$ : Find all 2-OPS which satisfy (2.2).

In this case, each $\left\{P_{n}\right\}_{n \geq 0}$ and $\left\{Q_{n}\right\}_{n \geq 0}$ satisfy a third-order recurrence relation which we write, respectively,

$$
\begin{array}{lc}
P_{n+3}(x)=\left(x-\beta_{n+2}\right) P_{n+2}(x)-\gamma_{n+2}^{1} P_{n+1}(x)-\gamma_{n+1}^{0} P_{n}(x), & \left(\gamma_{n+1}^{0} \neq 0\right), \\
& n \geq 0, \\
P_{0}(x)=1, \quad P_{1}(x)=x-\beta_{0}, P_{2}(x)=\left(x-\beta_{1}\right) P_{1}(x)-\gamma_{1}^{1}, &
\end{array}
$$

and

$$
\begin{array}{ll}
Q_{n+3}(x)=\left(x-\widetilde{\beta}_{n+2}\right) Q_{n+2}(x)-\widetilde{\gamma}_{n+2}^{1} Q_{n+1}(x)-\widetilde{\gamma}_{n+1}^{0} Q_{n}(x), & \begin{array}{c}
\left(\widetilde{\gamma}_{n+1}^{0} \neq 0\right), \\
n \geq 0,
\end{array} \\
Q_{0}(x)=1, \quad Q_{1}(x)=x-\widetilde{\beta}_{0}, \quad Q_{2}(x)=\left(x-\widetilde{\beta}_{1}\right) Q_{1}(x)-\widetilde{\gamma}_{1}^{1} . &
\end{array}
$$

First, it is easily seen that $P_{n}^{(1)}(x)=Q_{n}(x), n \geq 0$, if and only if

$$
\begin{array}{ll}
\widetilde{\beta}_{n}=\beta_{n+1}, & n \geq 0, \\
\widetilde{\gamma}_{n+1}^{1}=\gamma_{n+2}^{1}, & n \geq 0, \\
\widetilde{\gamma}_{n+1}^{0}=\gamma_{n+2}^{0}, & n \geq 0 .
\end{array}
$$

Next, by differentiating (2.14) and using the recurrences (2.14) and (2.15), we obtain that the sequences $\left\{\beta_{n}\right\},\left\{\widetilde{\beta}_{n}\right\},\left\{\gamma_{n}^{\nu}\right\}$, and $\left\{\widetilde{\gamma}_{n}^{\nu}\right\}(\nu=0,1)$ satisfy the system, valid for $n \geq 1[4]$,

$$
\begin{aligned}
& (n+2) \widetilde{\beta}_{n}-n \widetilde{\beta}_{n-1}=(n+1) \beta_{n+1}-(n-1) \beta_{n}, \\
& 2 \widetilde{\beta}_{0}=\beta_{0}+\beta_{1} \text {, } \\
& (n+3) \widetilde{\gamma}_{n+1}^{1}-(n+1) \widetilde{\gamma}_{n}^{1}=(n+1) \gamma_{n+2}^{1}-(n-1) \gamma_{n+1}^{1} \\
& +(n+1)\left(\beta_{n+1}-\widetilde{\beta}_{n}\right)^{2}, \\
& 3 \widetilde{\gamma}_{1}^{1}=\gamma_{2}^{1}+\gamma_{1}^{1}+\left(\beta_{1}-\widetilde{\beta}_{0}\right)^{2}, \\
& (n+4) \widetilde{\gamma}_{n+1}^{0}-(n+2) \widetilde{\gamma}_{n}^{0}=(n+1) \gamma_{n+2}^{0}-(n-1) \gamma_{n+1}^{0} \\
& +(n+1) \gamma_{n+2}^{1}\left(\beta_{n+2}+\beta_{n+1}-2 \widetilde{\beta}_{n}\right) \\
& -(n+2) \widetilde{\gamma}_{n+1}^{1}\left(2 \beta_{n+2}-\widetilde{\beta}_{n+1}-\widetilde{\beta}_{n}\right) \text {, } \\
& 4 \widetilde{\gamma}_{1}^{0}=\gamma_{2}^{0}+\gamma_{1}^{0}+\gamma_{2}^{1}\left(\beta_{2}+\beta_{1}-2 \widetilde{\beta}_{0}\right)-2 \widetilde{\gamma}_{1}^{1}\left(2 \beta_{2}-\widetilde{\beta}_{1}-\widetilde{\beta}_{0}\right) \text {, } \\
& n \gamma_{n+1}^{1} \gamma_{n+2}^{1}+(n+2) \widetilde{\gamma}_{n}^{1} \widetilde{\gamma}_{n+1}^{1}-2(n+1) \widetilde{\gamma}_{n}^{1} \gamma_{n+2}^{1} \\
& =(n+2) \widetilde{\gamma}_{n}^{0}\left(2 \beta_{n+2}-\widetilde{\beta}_{n+1}-\widetilde{\beta}_{n-1}\right) \\
& -n \gamma_{n+1}^{0}\left(\beta_{n+2}+\beta_{n}-2 \widetilde{\beta}_{n-1}\right), \\
& n \gamma_{n+1}^{0} \gamma_{n+3}^{0}=\widetilde{\gamma}_{n}^{0}\left(2(n+2) \gamma_{n+3}^{0}-(n+4) \widetilde{\gamma}_{n+2}^{0}\right) \text {, } \\
& n \gamma_{n+1}^{1} \gamma_{n+2}^{0}+n \gamma_{n+1}^{0} \gamma_{n+3}^{1}=\widetilde{\gamma}_{n}^{0}\left(2(n+2) \gamma_{n+3}^{1}-(n+3) \widetilde{\gamma}_{n+2}^{1}\right) \\
& +\widetilde{\gamma}_{n}^{1}\left(2(n+1) \gamma_{n+2}^{0}-(n+3) \widetilde{\gamma}_{n+1}^{0}\right) \text {. }
\end{aligned}
$$


From this system and making use (2.16), we obtain after some calculations that

$$
P_{n}^{(1)}(x)=Q_{n}(x), n \geq 0 \Longleftrightarrow \begin{cases}\beta_{n}=\beta_{0}, & n \geq 0, \\ \gamma_{n+1}^{1}=\frac{1}{2} \gamma_{1}^{1}, & n \geq 1, \\ \gamma_{n+1}^{0}=\frac{1}{3} \gamma_{1}^{0}, & n \geq 1 .\end{cases}
$$

We always can choose $\beta_{0}=0$ and $\gamma_{1}^{0}=3 \gamma \neq 0$ fixed, because orthogonality is kept through a shift. We put $\gamma_{1}^{1}=2 \alpha$, then the recurrence relations $(2.14)-(2.15)$ become, respectively,

$$
\begin{aligned}
P_{n+3}(x) & =x P_{n+2}(x)-\alpha P_{n+1}(x)-3^{\delta_{n, 0}} \gamma P_{n}(x), \quad n \geq 0, \\
P_{0}(x) & =1, \quad P_{1}(x)=x, \quad P_{2}(x)=x^{2}-2 \alpha,
\end{aligned}
$$

and

$$
\begin{aligned}
Q_{n+3}(x) & =x Q_{n+2}(x)-\alpha Q_{n+1}(x)-\gamma Q_{n}(x), \quad n \geq 0, \\
Q_{0}(x) & =1, \quad Q_{1}(x)=x, \quad Q_{2}(x)=x^{2}-\alpha .
\end{aligned}
$$

It is clear that $\left\{Q_{n}\right\}_{n \geq 0}$ is the classical 2-orthogonal polynomial sequence found in Part I, say, the Tchebychev 2-OPS of the second kind.

Let us now introduce the generating function for the polynomials $P_{n}, n \geq 0$.

$$
F(x, t)=\sum_{n \geq 0} P_{n}(x) t^{n}
$$

By virtue of (2.26), we easily obtain

$$
F(x, t)=\frac{1-\alpha t^{2}-2 \gamma t^{3}}{1-x t+\alpha t^{2}+\gamma t^{3}} .
$$

Note that $F(x, t)=\left(1-\alpha t^{2}-2 \gamma t^{3}\right) G(x, t)$ where $G(x, t)=\left(1-x t+\alpha t^{2}+\gamma t^{3}\right)^{-1}$ is the generating function of the 2-orthogonal Tchebychev polynomials of the second kind, $\left\{Q_{n}\right\}_{n \geq 0}$.

Remark 2.2. The polynomials obtained here appear as particular cases of Faber polynomials [9]. Such polynomials are defined as follows: let $z$ and $w$ be two complex variables, $f(z)$ be a mapping, and $h(w)$ be its inverse.

$$
\begin{aligned}
& w=f(z)=z+a_{0}+a_{1} z^{-1}+a_{2} z^{-2}+\cdots, \\
& z=h(w)=w+b_{0}+b_{1} w^{-1}+b_{2} w^{-2}+\ldots,
\end{aligned}
$$

$f(z)$ is a univalent mapping from a complement of a compact set onto a domain $|w|>r$ ( $r$ is the smallest number such that $h(w)$ is univalent in $|w|>r$ ).

The Faber polynomials $F_{n}(z)$ of degree $n$, associated with $f(z)$, are the polynomial parts of $[f(z)]^{n}$. Many properties of the polynomials $F_{n}$ are known. We have, for example, the generating function

$$
\frac{w h^{\prime}(w)}{h(w)-z}=\sum_{n \geq 0} F_{n}(z) w^{-n},
$$

which holds for $|w|$ sufficiently large (depending on $z$ ). The relation (2.29) often is taken as the definition of Faber polynomials. 
If we put $t=1 / w$, the above becomes

$$
\frac{h^{\prime}(1 / t)}{t h(1 / t)}\left\{1-\frac{z}{h(1 / t)}\right\}^{-1}=\sum_{n \geq 0} F_{n}(z) t^{n} .
$$

(1) It is easy to verify that the choice $h(w)=\left(w^{2}+1\right) / 2 w$ leads to the Tchebychev non-monic polynomials of the first kind $\left\{T_{n}\right\}_{n \geq 0}$.

(2) Similarly, the choice $h(w)=\left(w^{3}+\alpha w+\gamma\right) / w^{2}$ leads to the 2-OPS $\left\{P_{n}\right\}_{n \geq 0}$ defined by the recurrence (2.26).

Indeed, in (2.29), the substitution of $h(w)=\left(w^{3}+\alpha w+\gamma\right) / w^{2}$, with $t=1 / w$, leads to

$$
\frac{h^{\prime}(1 / t)}{t h(1 / t)}\left\{1-\frac{x}{h(1 / t)}\right\}^{-1}=\sum_{n \geq 0} F_{n}(x) t^{n}
$$

with

$$
\frac{h^{\prime}(1 / t)}{t h(1 / t)}\left\{1-\frac{x}{h(1 / t)}\right\}^{-1}=\frac{1-\alpha t^{2}-2 \gamma t^{3}}{1-x t+\alpha t^{2}+\gamma t^{3}}=F(x, t) .
$$

Then $F_{n}(x)=P_{n}(x), n \geq 0$.

2.2. 2-Symmetric polynomials and cubic decomposition. Let $k$ be an integer with $0 \leq k \leq d$. By $\xi_{k}, k=0,1, \ldots, d$, we denote the $d+1$ roots of unity, namely,

$$
\xi_{k}=\exp \left(\frac{2 i k \pi}{d+1}\right) .
$$

Definition 2.1. The sequence $\left\{P_{n}\right\}_{n \geq 0}$ is called $d$-symmetric if it fulfills

$$
P_{n}\left(\xi_{k} x\right)=\xi_{k}^{n} P_{n}(x), \quad k=0,1, \ldots, d, \quad n \geq 0 .
$$

When $d=1$, then $\xi_{0}=1$ and $\xi_{1}=-1$; this means that the sequence $\left\{P_{n}\right\}_{n \geq 0}$ is symmetric, that is, $P_{n}(-x)=(-1)^{n} P_{n}(x), n \geq 0$.

In order to produce the 2-OPS analogous to the Tchebychev OPS of the first kind, we put $\alpha=0$. Hence, the 2-OPS $\left\{P_{n}\right\}_{n \geq 0}$ is 2 -symmetric. In this case, $\left\{P_{n}\right\}_{n \geq 0}$ is the "classical" 2-OPS analogous to the Tchebychev OPS of the first kind. Therefore, they will be called the Tchebychev 2-OPS of the first kind.

When $\alpha=0$, the recurrence relation $(2.26)$ becomes

$$
\begin{gathered}
P_{n+3}(x)=x P_{n+2}(x)-3^{\delta_{n, 0}} \gamma P_{n}(x), \quad n \geq 0, \\
P_{0}(x)=1, \quad P_{1}(x)=x, \quad P_{2}(x)=x^{2} .
\end{gathered}
$$

Hence, setting

$$
\begin{aligned}
P_{3 n}(x) & =P_{n}^{[1]}\left(x^{3}\right), \\
P_{3 n+1}(x) & =x P_{n}^{[2]}\left(x^{3}\right), \\
P_{3 n+2}(x) & =x^{2} P_{n}^{[3]}\left(x^{3}\right), \quad n \geq 0,
\end{aligned}
$$




\begin{tabular}{|c|c|c|c|}
\hline & ${ }^{i} \beta_{n}$ & ${ }^{i} \gamma_{n+1}^{1}$ & ${ }^{i} \gamma_{n+1}^{0}$ \\
\hline$i=1$ & $3 \gamma$ & $3 \gamma^{2}$ except ${ }^{1} \gamma_{1}^{1}=6 \gamma^{2}$ & $\gamma^{3}$ except: ${ }^{1} \gamma_{1}^{0}=3 \gamma^{3}$ \\
\hline$i=2$ & $3 \gamma$ except: ${ }^{2} \beta_{0}=4 \gamma$ & $3 \gamma^{2}$ except: ${ }^{2} \gamma_{1}^{1}=5 \gamma^{2}$ & $\gamma^{3}$ \\
\hline$i=3$ & $3 \gamma$ except: ${ }^{3} \beta_{0}=5 \gamma$ & $3 \gamma^{2}$ & $\gamma^{3}$ \\
\hline
\end{tabular}

TABLE 1. Recurrence coefficients

where $\left\{P_{n}^{[i]}\right\}_{n \geq 0}, i=1,2,3$, are the three components of $\left\{P_{n}\right\}_{n \geq 0}$ which are given by the system [4]

$$
\left(\begin{array}{l}
P_{n+1}^{[1]}(x) \\
P_{n+1}^{[2]}(x) \\
P_{n+1}^{[3]}(x)
\end{array}\right)=\left(\begin{array}{ccc}
-\gamma & 0 & x \\
-\gamma & -\gamma & x \\
-\gamma & -\gamma & x-\gamma
\end{array}\right)\left(\begin{array}{l}
P_{n}^{[1]}(x) \\
P_{n}^{[2]}(x) \\
P_{n}^{[3]}(x)
\end{array}\right), \quad n \geq 1,
$$

with $P_{0}^{[1]}(x)=P_{0}^{[2]}(x)=P_{0}^{[3]}(x)=1$ and $P_{1}^{[1]}(x)=x-3 \gamma, P_{1}^{[2]}(x)=x-4 \gamma$, $P_{1}^{[3]}(x)=x-5 \gamma$.

This natural (cubic) decomposition produces other sequences which are also 2-orthogonal. In particular, the first component $\left\{P_{n}^{[1]}\right\}_{n \geq 0}$ is a "classical" one.

It follows that each $\left\{P_{n}^{[i]}\right\}_{n \geq 0},(i=1,2,3)$ satisfies a third-order recurrence relation (see [4] for the formulas)

$$
\begin{gathered}
P_{n+3}^{[i]}(x)=\left(x-{ }^{i} \beta_{n+2}\right) P_{n+2}^{[i]}(x)-{ }^{i} \gamma_{n+2}^{1} P_{n+1}^{[i]}(x)-{ }^{i} \gamma_{n+1}^{0} P_{n}^{[i]}(x),\left({ }^{i} \gamma_{n+1}^{0} \neq 0\right), \\
n \geq 0, \\
P_{0}^{[i]}(x)=1, \quad P_{1}^{[i]}(x)=x-{ }^{i} \beta_{0}, \quad P_{2}^{[i]}(x)=\left(x-{ }^{i} \beta_{1}\right) P_{1}^{[i]}(x)-{ }^{i} \gamma_{1}^{1},
\end{gathered}
$$

where the recurrence coefficients ${ }^{i} \beta_{n},{ }^{i} \gamma_{n+1}^{1}$, and ${ }^{i} \gamma_{n+1}^{0}, n \geq 0$, are tabulated in Table 1 , and for each $i=1,2,3$, the sequence $\left\{P_{n}^{[i]}\right\}_{n \geq 0}$ is 2-orthogonal with respect to $\mathcal{U}^{[i]}={ }^{t}\left(u_{0}^{[i]}, u_{1}^{[i]}\right)$ where, according to the relation (2.18) given in Part I [6]

$$
u_{j}^{[i]}=\sigma_{3}\left(x^{i-1} u_{3 j+i-1}\right), \quad j=0,1, i=1,2,3 .
$$

The cubic decomposition of the sequence $\left\{Q_{n}\right\}_{n \geq 0}$ was made in Part I [6].

\section{A differential-recurrence relation and a third-order differential equation}

We start by proving that the sequence $\left\{P_{n}\right\}_{n \geq 0}$ defined by the generating function (2.28) satisfies a first-order differential-recurrence relation. Next, when $\alpha=0$, we give a third-order differential equation for the polynomials $P_{n}, n \geq 0$.

Proposition 3.1. The polynomial sequence $\left\{P_{n}\right\}_{n \geq 0}$ satisfies the differential-recurrence relation

$$
\begin{aligned}
& \phi(x) P_{n+1}^{\prime}(x)=(n+1) A_{1}(x) P_{n+1}(x)+\left[n A_{2}(x)+B_{1}(x)\right] P_{n}(x) \\
& \quad+\left[(n-1) A_{3}(x)+B_{2}(x)\right] P_{n-1}(x)+\left[(n-2) A_{4}(x)+B_{3}(x)\right] P_{n-2}(x) \\
& \quad+\left[(n-3) A_{5}(x)+B_{4}(x)\right] P_{n-3}(x)+(n-4) A_{6}(x) P_{n-4}(x), \quad n \geq 0,
\end{aligned}
$$


where $\phi(x)=\left(4 \alpha \gamma x+\alpha^{3}+9 \gamma^{2}\right)\left(4 \gamma x^{3}+\alpha^{2} x^{2}-18 \alpha \gamma x-4 \alpha^{3}-27 \gamma^{2}\right)$ and $A_{i}(x)$, $1 \leq i \leq 6, B_{j}(x), 1 \leq j \leq 4$, are polynomials of degree at most equal to 3 (see the equalities (3.12), (3.13) below). We have put $P_{-n}(x)=0, n \geq 1$.

Proof. From the generating function (2.28), we obtain by differentiating $F(x, t)$ with respect to $t$ and $x$, respectively,

$$
\begin{gathered}
\left(2 \gamma t^{3}+\alpha t^{2}-1\right)\left(\gamma t^{3}+\alpha t^{2}-x t+1\right) \partial_{t} F(x, t) \\
=\left[\alpha \gamma t^{4}-4 \gamma x t^{3}+(9 \gamma-\alpha x) t^{2}+4 \alpha t-x\right] F(x, t) \\
\left(\gamma t^{3}+\alpha t^{2}-x t+1\right) \partial_{x} F(x, t)=t F(x, t)
\end{gathered}
$$

where $\partial_{x}=\partial / \partial x$ and $\partial_{t}=\partial / \partial t$.

From these, it follows that

$$
\left[\alpha \gamma t^{4}-4 \gamma x t^{3}+(9 \gamma-\alpha x) t^{2}+4 \alpha t-x\right] \partial_{x} F(x, t)=\left(2 \gamma t^{4}+\alpha t^{3}-t\right) \partial_{t} F(x, t) \text {. }
$$

Next, multiplying (3.3) by $-\alpha t$ and adding to (3.4), we obtain

$$
\begin{aligned}
{\left[\left(4 \gamma x+\alpha^{2}\right) t^{3}-9 \gamma t^{2}-3 \alpha t+x\right] \partial_{x} F(x, t)=- } & \left(2 \gamma t^{4}+\alpha t^{3}-t\right) \partial_{t} F(x, t) \\
& +\alpha t^{2} F(x, t) .
\end{aligned}
$$

Now multiplying (3.5) by $-\gamma$ and (3.3) by $4 \gamma x+\alpha^{2}$ and adding, we obtain

$$
\begin{gathered}
{\left[-\left(4 \alpha \gamma x+\alpha^{3}+9 \gamma^{2}\right) t^{2}+\left(4 \gamma x^{2}+\alpha^{2} x-3 \alpha \gamma\right) t-\left(3 \gamma x+\alpha^{2}\right)\right] \partial_{x} F(x, t)} \\
=-\gamma\left(2 \gamma t^{4}+\alpha t^{3}-t\right) \partial_{t} F(x, t)+\left[\alpha \gamma t^{2}-\left(4 \gamma x+\alpha^{2}\right) t\right] F(x, t) .
\end{gathered}
$$

Likewise, multiplying (3.6) by $\gamma t$ and (3.3) by $4 \alpha \gamma x+\alpha^{2}+9 \gamma^{2}$, we obtain by addition

$$
\begin{gathered}
{\left[\left(4 \gamma^{2} x^{2}+5 \alpha^{2} \gamma x+6 \alpha \gamma^{2}+\alpha^{4}\right) t^{2}-\left(4 \alpha \gamma x^{2}+\left(\alpha^{3}+12 \gamma^{2}\right) x+\alpha^{2} \gamma\right) t\right.} \\
\left.\quad+\left(4 \alpha \gamma x+\alpha^{3}+9 \gamma^{2}\right)\right] \partial_{x} F(x, t) \\
=-\gamma^{2}\left(2 \gamma t^{5}+\alpha t^{4}-t^{2}\right) \partial_{t} F(x, t) \\
\quad+\left[\alpha \gamma^{2} t^{3}-\gamma\left(4 \gamma x+\alpha^{2}\right) t^{2}+\left(4 \alpha \gamma x+\alpha^{3}+9 \gamma^{2}\right) t\right] F(x, t)
\end{gathered}
$$

Thus, (3.6) and (3.7) form the system

$$
\begin{aligned}
& m_{11}(x) t^{2} \partial_{x} F(x, t)+m_{12}(x) t \partial_{x} F(x, t)=n_{1}(x, t), \\
& m_{21}(x) t^{2} \partial_{x} F(x, t)+m_{22}(x) t \partial_{x} F(x, t)=n_{2}(x, t)
\end{aligned}
$$

where

$$
\begin{array}{ll}
m_{11}(x)=4 \alpha \gamma x+9 \gamma^{2}+\alpha^{3}, & m_{12}(x)=-4 \gamma x^{2}-\alpha^{2} x+3 \alpha \gamma \\
m_{21}(x)=4 \gamma^{2} x^{2}+5 \alpha^{2} \gamma x+6 \alpha \gamma^{2}+\alpha^{4}, & m_{22}(x)=-4 \alpha \gamma x^{2}-\left(\alpha^{3}+12 \gamma^{2}\right) x-\alpha^{2} \gamma
\end{array}
$$

and

$$
\begin{aligned}
n_{1}(x, t)=- & \left(3 \gamma x+\alpha^{2}\right) \partial_{x} F(x, t)+\gamma\left(2 \gamma t^{4}+\alpha t^{3}-t\right) \partial_{t} F(x, t) \\
& +\left[-\alpha \gamma t^{2}+\left(4 \gamma x+\alpha^{2}\right) t\right] F(x, t), \\
n_{2}(x, t)=- & \left(4 \alpha \gamma x+\alpha^{3}+9 \gamma^{2}\right) \partial_{x} F(x, t)-\gamma^{2}\left(2 \gamma t^{5}+\alpha t^{4}-t^{2}\right) \partial_{t} F(x, t) \\
& +\left[\alpha \gamma^{2} t^{3}-\gamma\left(4 \gamma x+\alpha^{2}\right) t^{2}+\left(4 \alpha \gamma x+\alpha^{3}+9 \gamma^{2}\right) t\right] F(x, t) .
\end{aligned}
$$

Let $\Delta(x)$ be the determinant of the above system and $\delta(x)$ the polynomial given by

$$
\delta(x)=4 \gamma x^{3}+\alpha^{2} x^{2}-18 \alpha \gamma x-4 \alpha^{3}-27 \gamma^{2} .
$$


We have $\Delta(x)=m_{11}(x) m_{22}(x)-m_{21}(x) m_{12}(x)=\gamma\left(4 \gamma x+\alpha^{2}\right) \delta(x)$. as

Hence, the system (3.8) allows us to express $t^{2} \partial_{x} F(x, t)$ and $t \partial_{x} F(x, t)$, respectively,

$$
\begin{array}{r}
\Delta(x) t^{2} \partial_{x} F(x, t)=\left[a_{5}(x) t^{5}+a_{4}(x) t^{4}+a_{3}(x) t^{3}+a_{2}(x) t^{2}+a_{1}(x) t\right] \partial_{t} F(x, t) \\
+\left[b_{3}(x) t^{3}+b_{2}(x) t^{2}+b_{1}(x) t\right] F(x, t)-\alpha \gamma \delta(x) \partial_{x} F(x, t)
\end{array}
$$

where

$$
\begin{aligned}
& a_{1}(x)=4 \alpha \gamma^{2} x^{2}+\gamma\left(\alpha^{3}+12 \gamma^{2}\right) x+\alpha^{2} \gamma^{2}, \\
& a_{2}(x)=4 \gamma^{3} x^{2}+\alpha^{2} \gamma^{2} x-3 \alpha \gamma^{3} \\
& a_{3}(x)=-4 \alpha^{2} \gamma^{2} x^{2}-\alpha \gamma\left(\alpha^{3}+12 \gamma^{2}\right) x-\alpha^{3} \gamma^{2}, \\
& a_{4}(x)=-12 \alpha \gamma^{3} x^{2}-3 \gamma^{2}\left(\alpha^{3}+8 \gamma^{2}\right) x+\alpha^{2} \gamma^{3} \\
& a_{5}(x)=-8 \gamma^{4} x^{2}-2 \alpha^{2} \gamma^{3} x+6 \alpha \gamma^{4} \\
& b_{1}(x)=-12 \gamma^{3} x^{2}-19 \alpha^{2} \gamma^{2} x-\alpha \gamma\left(4 \alpha^{3}+27 \gamma^{2}\right), \\
& b_{2}(x)=-16 \gamma^{3} x^{3}-4 \alpha^{2} \gamma^{2} x^{2}+24 \alpha \gamma^{3} x+4 \alpha^{3} \gamma^{2}, \\
& b_{3}(x)=4 \alpha \gamma^{3} x^{2}+\alpha^{3} \gamma^{2} x-3 \alpha^{2} \gamma^{3}
\end{aligned}
$$

and

$$
\begin{aligned}
\Delta(x) t \partial_{x} F(x, t)=[ & \left.c_{5}(x) t^{5}+c_{4}(x) t^{4}+c_{3}(x) t^{3}+c_{2}(x) t^{2}+c_{1}(x) t\right] \partial_{t} F(x, t) \\
& +\left[d_{3}(x) t^{3}+d_{2}(x) t^{2}+d_{1}(x) t\right] F(x, t)+3 \gamma^{2} \delta(x) \partial_{x} F(x, t)
\end{aligned}
$$

where

$$
\begin{aligned}
& c_{1}(x)=4 \gamma^{3} x^{2}+5 \alpha^{2} \gamma^{2} x+\alpha \gamma\left(\alpha^{3}+6 \gamma^{2}\right) \\
& c_{2}(x)=4 \alpha \gamma^{3} x+\gamma^{2}\left(\alpha^{3}+9 \gamma^{2}\right) \\
& c_{3}(x)=-4 \alpha \gamma^{3} x^{2}-5 \alpha^{3} \gamma^{2} x-\alpha^{2} \gamma\left(\alpha^{3}+6 \gamma^{2}\right) \\
& c_{4}(x)=-8 \gamma^{4} x^{2}-14 \alpha^{2} \gamma^{3} x-3 \alpha \gamma^{2}\left(\alpha^{3}+7 \gamma^{2}\right) \\
& c_{5}(x)=-8 \alpha \gamma^{4} x-2 \gamma^{3}\left(\alpha^{3}+9 \gamma^{2}\right) \\
& d_{1}(x)=-16 \gamma^{3} x^{3}-8 \alpha^{2} \gamma^{2} x^{2}-\alpha \gamma\left(\alpha^{3}-48 \gamma^{2}\right) x+3 \gamma^{2}\left(4 \alpha^{3}+27 \gamma^{2}\right) \\
& d_{2}(x)=-12 \alpha \gamma^{3} x^{2}-3 \gamma^{2}\left(\alpha^{3}+12 \gamma^{2}\right) x-3 \alpha^{2} \gamma^{3} \\
& d_{3}(x)=4 \alpha^{2} \gamma^{3} x+\alpha \gamma^{2}\left(\alpha^{3}+9 \gamma^{2}\right)
\end{aligned}
$$

Now, by multiplying the equality (3.10) by $t$, using (3.9) and (3.10) again, we obtain after some calculations

$$
\phi(x) \partial_{x} F(x, t)=A(x, t) \partial_{t} F(x, t)+B(x, t) F(x, t)
$$

where

$$
\phi(x)=\left(4 \alpha \gamma x+\alpha^{3}+9 \gamma^{2}\right) \delta(x), A(x, t)=\sum_{\nu=1}^{6} A_{\nu}(x) t^{\nu}, B(x, t)=\sum_{\nu=1}^{4} B_{\nu}(x) t^{\nu},
$$


with

$$
\begin{aligned}
& A_{1}(x)=16 \alpha \gamma^{2} x^{3}+4 \gamma\left(2 \alpha^{3}+9 \gamma^{2}\right) x^{2}+\alpha^{2}\left(\alpha^{3}+\gamma^{2}\right) x-2 \alpha \gamma\left(\alpha^{3}+9 \gamma^{2}\right), \\
& A_{2}(x)=-16 \alpha^{2} \gamma^{2} x^{2}-8 \alpha \gamma\left(\alpha^{3}+6 \gamma^{2}\right) x-\left(\alpha^{3}+3 \gamma^{2}\right)\left(\alpha^{3}+9 \gamma^{2}\right) \text {, } \\
& A_{3}(x)=-16 \alpha^{2} \gamma^{2} x^{3}-4 \alpha \gamma\left(2 \alpha^{3}+13 \gamma^{2}\right) x^{2}-\left(\alpha^{6}+9 \gamma^{2}\left(\alpha^{3}+4 \gamma^{2}\right)\right) x \\
& +\alpha^{2} \gamma\left(\alpha^{3}+9 \gamma^{2}\right) \\
& A_{4}(x)=-32 \alpha \gamma^{3} x^{3}-72 \gamma^{4} x^{2}+2 \alpha^{2} \gamma\left(3 \alpha^{3}+23 \gamma^{2}\right) x+\alpha\left(\alpha^{3}+7 \gamma^{2}\right)\left(\alpha^{3}+9 \gamma^{2}\right) \text {, } \\
& A_{5}(x)=48 \alpha^{2} \gamma^{3} x^{2}+12 \alpha \gamma^{2}\left(2 \alpha^{3}+11 \gamma^{2}\right) x+3 \gamma\left(\alpha^{3}+2 \gamma^{2}\right)\left(\alpha^{3}+9 \gamma^{2}\right) \text {, } \\
& A_{6}(x)=32 \alpha \gamma^{4} x^{2}+8 \gamma^{3}\left(2 \alpha^{3}+9 \gamma^{2}\right) x+2 \alpha^{2} \gamma^{2}\left(\alpha^{3}+9 \gamma^{2}\right) \text {, }
\end{aligned}
$$

and

$$
\begin{aligned}
& B_{1}(x)=-64 \alpha^{2} \gamma^{2} x^{2}-4 \alpha \gamma\left(8 \alpha^{3}+63 \gamma^{2}\right) x-\left(\alpha^{3}+9 \gamma^{2}\right)\left(4 \alpha^{3}+27 \gamma^{2}\right), \\
& B_{2}(x)=16 \alpha^{2} \gamma^{2} x^{3}+4 \alpha \gamma\left(2 \alpha^{3}-15 \gamma^{2}\right) x^{2}+\left(\alpha^{6}-\gamma^{2}\left(47 \alpha^{3}+216 \gamma^{2}\right)\right) x \\
& \quad-8 \alpha^{2} \gamma\left(\alpha^{3}+9 \gamma^{2}\right), \\
& B_{3}(x)=64 \alpha \gamma^{3} x^{3}+16 \gamma^{2}\left(2 \alpha^{3}+9 \gamma^{2}\right) x^{2}+4 \alpha^{2} \gamma\left(\alpha^{3}+6 \gamma^{2}\right) x-3 \alpha \gamma^{2}\left(\alpha^{3}+9 \gamma^{2}\right), \\
& B_{4}(x)=-16 \alpha^{2} \gamma^{3} x^{2}-4 \alpha \gamma^{2}\left(2 \alpha^{3}+9 \gamma^{2}\right) x-\alpha^{3} \gamma\left(\alpha^{3}+9 \gamma^{2}\right) .
\end{aligned}
$$

Hence, on substituting the series expansion for $F(x, t)$ into (3.11), we find the relation (3.1).

In the particular case when $\alpha=0$, the differential-recurrence relation (3.1) becomes

$$
\begin{aligned}
\left(x^{3}-\frac{27}{4} \gamma\right) P_{n+1}^{\prime}(x)= & (n+1) x^{2} P_{n+1}(x)-\frac{3}{4} \gamma(n+9) P_{n}(x) \\
& -\gamma(n+5) x P_{n-1}(x)-2 \gamma(n-4) x^{2} P_{n-2}(x) \\
& +\frac{3}{2} \gamma^{2}(n-3) x P_{n-3}(x)+2 \gamma^{2}(n-4) x P_{n-4}(x), \quad n \geq 0 .
\end{aligned}
$$

Using the recurrence relation (2.30), the above equation becomes

$$
\begin{aligned}
\left(x^{3}-\frac{27}{4} \gamma\right) P_{n+1}^{\prime}(x)=( & +1) P_{n+3}(x)-\frac{7}{4} \gamma(n+1) P_{n}(x) \\
& -\frac{1}{2} \gamma^{2}(n+1) P_{n-3}(x), \quad n \geq 3,
\end{aligned}
$$

with the initial conditions $P_{1}^{\prime}(x)=P_{0}(x)=1, P_{2}^{\prime}(x)=2 P_{1}(x)=2 x, P_{3}^{\prime}(x)=$ $3 P_{2}(x)=3 x^{2}$. Note that, by differentiating this equation, we obtain the relation (3.10) given in Part I.

Proposition 3.2. Let $\left\{P_{n}\right\}_{n \geq 0}$ be a 2-symmetric sequence of 2-orthogonal polynomials. If $\left\{P_{n}\right\}_{n \geq 0}$ is 2-classical, then each polynomial $P_{n}$ satisfies a third-order differential equation of the form

$$
\left(a_{n} x^{3}-b_{n}\right) P_{n}^{\prime \prime \prime}(x)+c_{n} x^{2} P_{n}^{\prime \prime}(x)+d_{n} x P_{n}^{\prime}(x)-e_{n} P_{n}(x)=0, \quad n \geq 0,
$$


where

$$
\begin{aligned}
& a_{n}=\left(\vartheta_{n}-1\right)\left(\vartheta_{n+1}-1\right), \\
& n \geq 1 \quad\left(a_{0}=0\right), \\
& b_{n}=\frac{\gamma_{n+3}^{0}}{(n+3)(n+4)}\left[(n+3) \vartheta_{n}-(n+2)\right] \\
& \times\left[(n+4) \vartheta_{n+1}-(n+3)\right]\left[(n+5) \vartheta_{n+2}-(n+4)\right], \quad n \geq 1 \quad\left(b_{0}=0\right), \\
& c_{n}=\vartheta_{n} \vartheta_{n+1}-1-(n-3)\left(\vartheta_{n}-1\right)\left(\vartheta_{n+1}-1\right) \text {, } \\
& n \geq 1 \quad\left(c_{0}=0\right) \text {, } \\
& d_{n}=n \vartheta_{n+1}-(n-1) \vartheta_{n}\left(2 \vartheta_{n+1}-1\right) \text {, } \\
& n \geq 1 \quad\left(d_{0}=0\right), \\
& e_{n}=n \vartheta_{n+1} \text {, } \\
& n \geq 0 \text {, }
\end{aligned}
$$

with $\vartheta_{n}=(n+2) \widetilde{\gamma}_{n}^{0}\left(n \gamma_{n+1}^{0}\right)^{-1}, n \geq 1$.

Proof. From the hypothesis, the sequence $\left\{P_{n}\right\}_{n \geq 0}$ satisfies the recurrence

$$
\begin{aligned}
P_{n+3}(x) & =x P_{n+2}(x)-\gamma_{n+1}^{0} P_{n}(x), \quad n \geq 0, \\
P_{0}(x) & =1, \quad P_{1}(x)=x, \quad P_{2}(x)=x^{2},
\end{aligned}
$$

and the sequence $\left\{Q_{n}\right\}_{n \geq 0}$, defined by $Q_{n}(x)=P_{n+1}^{\prime}(x) /(n+1)$, also satisfies

$$
\begin{aligned}
Q_{n+3}(x) & =x Q_{n+2}(x)-\widetilde{\gamma}_{n+1}^{0} Q_{n}(x), \quad n \geq 0, \\
Q_{0}(x) & =1, \quad Q_{1}(x)=x, \quad Q_{2}(x)=x^{2}
\end{aligned}
$$

where $\widetilde{\gamma}_{n}^{0}=\frac{n}{n+2} \gamma_{n+1}^{0} \vartheta_{n}, n \geq 1$.

First, by differentiating (3.15) and using (3.16), we obtain, after changing $n$ to $n+1$

$$
\begin{aligned}
P_{n+3}(x) & =Q_{n+3}(x)-k_{n+1} Q_{n}(x), \quad n \geq 0, \\
P_{0}(x) & =Q_{0}(x)=1, \quad P_{1}(x)=Q_{1}(x)=x, \quad P_{2}(x)=Q_{2}(x)=x^{2}
\end{aligned}
$$

where $k_{n}=(n+2) \widetilde{\gamma}_{n}^{0}-n \gamma_{n+1}^{0}=n \gamma_{n+1}^{0}\left(\vartheta_{n}-1\right), n \geq 1$.

By differentiating (3.17), we get

$$
(n+3) Q_{n+2}(x)=Q_{n+3}^{\prime}(x)-k_{n+1} Q_{n}^{\prime}(x), \quad n \geq 0 .
$$

From this and using the derivative of (3.16), we obtain

$$
(n+2) Q_{n+2}(x)=x Q_{n+2}^{\prime}(x)-l_{n+1} Q_{n}^{\prime}(x), \quad n \geq 0,
$$

where $l_{n}=k_{n}+\widetilde{\gamma}_{n}^{0}=\frac{n}{n+2} \gamma_{n+1}^{0}\left((n+3) \vartheta_{n}-(n+2)\right), n \geq 1$.

Next, differentiating (3.18), we get

$$
(n+1) Q_{n+2}^{\prime}(x)=x Q_{n+2}^{\prime \prime}(x)-l_{n+1} Q_{n}^{\prime \prime}(x), \quad n \geq 0 .
$$

Further, from (3.1), (3.15), and (3.16), after changing $n$ to $n+1$, we obtain

$$
x Q_{n+3}(x)-l_{n+2} Q_{n+1}(x)=x P_{n+3}(x)-\gamma_{n+2}^{0} P_{n+1}, \quad n \geq 0 .
$$

Multiplying (3.16) by $x$ and using (3.20), we have

$$
\gamma_{n+2}^{0} P_{n+1}(x)=l_{n+2} Q_{n+1}(x)-k_{n+1} x Q_{n}(x), \quad n \geq 0,
$$

and by changing $n$ to $n+1$, we get

$$
\gamma_{n+3}^{0} P_{n+2}(x)=l_{n+3} Q_{n+2}(x)-k_{n+2} x Q_{n+1}(x), \quad n \geq 0 .
$$

Now, differentiating the above, we obtain

$$
l_{n+3} Q_{n+2}^{\prime}(x)=\left[(n+2) \gamma_{n+3}^{0}+k_{n+2}\right] Q_{n+1}(x)+k_{n+2} x Q_{n+1}^{\prime}(x), \quad n \geq 0
$$


Multiply (3.23) by $l_{n+2}$, use (3.21) and its derivative to get

$$
\begin{array}{r}
\frac{l_{n+2} l_{n+3}}{(n+2) \gamma_{n+2}^{0} \gamma_{n+3}^{0}} Q_{n+2}^{\prime}(x)=\vartheta_{n+2} P_{n+1}(x)+\left(2 \vartheta_{n+1} \vartheta_{n+2}-\vartheta_{n+1}-\vartheta_{n+2}\right) x P_{n+1}^{\prime}(x) \\
+\left(\vartheta_{n+1}-1\right)\left(\vartheta_{n+2}-1\right) x^{2} P_{n+1}^{\prime \prime}(x), \quad n \geq 0 . \quad
\end{array}
$$

We let

$$
\begin{aligned}
\alpha_{n} & =\frac{l_{n+1} l_{n+2}}{(n+1) \gamma_{n+1}^{0} \gamma_{n+2}^{0}}, \quad n \geq 0, \\
\delta_{n} & =2 \vartheta_{n} \vartheta_{n+1}-\vartheta_{n}-\vartheta_{n+1}, \quad n \geq 1 \quad\left(\delta_{0}=0\right), \\
\zeta_{n} & =\left(\vartheta_{n}-1\right)\left(\vartheta_{n+1}-1\right), \quad n \geq 1 \quad\left(\zeta_{0}=0\right) .
\end{aligned}
$$

Then, the relation (3.24) can be written as

$$
\alpha_{n+1} Q_{n+2}^{\prime}(x)=\zeta_{n+1} x^{2} P_{n+1}^{\prime \prime}(x)+\delta_{n+1} x P_{n+1}^{\prime}(x)+\vartheta_{n+2} P_{n+1}(x), \quad n \geq 0,
$$

which gives upon differentiation

$$
\begin{gathered}
\alpha_{n+1} Q_{n+2}^{\prime \prime}(x)=\zeta_{n+1} x^{2} P_{n+1}^{\prime \prime \prime}(x)+\left(\delta_{n+1}+2 \zeta_{n+1}\right) x P_{n+1}^{\prime \prime}(x) \\
+\left(\delta_{n+1}+\vartheta_{n+2}\right) P_{n+1}^{\prime}(x), \quad n \geq 0
\end{gathered}
$$

Now, from (3.19) and using the relations (3.25) and (3.26), we easily obtain

$$
\begin{aligned}
& \left(\zeta_{n+1} x^{3}-(n+1)^{-1} l_{n+1} \alpha_{n+1}\right) P_{n+1}^{\prime \prime \prime}(x)+\left(\delta_{n+1}-(n-1) \zeta_{n+1}\right) x^{2} P_{n+1}^{\prime \prime}(x) \\
& \quad+\left(\vartheta_{n+2}-n \delta_{n+1}\right) x P_{n+1}^{\prime}(x)-(n+1) \vartheta_{n+2} P_{n+1}(x)=0, \quad n \geq 0 .
\end{aligned}
$$

After some simplifications and by changing $n$ to $n-1$, the third-order differential equation (3.14) follows immediately.

Application. The above result for the Tchebychev 2-orthogonal polynomials $\left\{P_{n}\right\}_{n \geq 0}$ defined by the recurrence (2.30) is adapted to our situation. We obtain the third-order differential equation satisfied by each polynomial $P_{n}, n=0,1, \ldots$,

$$
\begin{aligned}
& 4\left(x^{3}-1\right) P_{n}^{\prime \prime \prime}(x)+18 x^{2} P_{n}^{\prime \prime}(x) \\
& \quad-\left(3 n^{2}+3 n-10\right) x P_{n}^{\prime}(x)-n^{2}(n+3) P_{n}(x)=0, \quad n \geq 0 .
\end{aligned}
$$

Indeed, in this case, we have $\gamma_{n+1}^{0}=\gamma=4 / 27, n \geq 1, \gamma_{1}^{0}=3 \gamma, \widetilde{\gamma}_{n+1}^{0}=\gamma, n \geq 0$, and $\vartheta_{n}=(n+2) / n, n \geq 1$; thus the coefficients $a_{n}, b_{n}, c_{n}, d_{n}$, and $e_{n}$ are given by

$$
\begin{array}{ll}
a_{n}=\frac{4}{n(n+1)}, & n \geq 1\left(a_{0}=0\right), \\
b_{n}=\frac{27 \gamma}{n(n+1)}, & n \geq 1\left(b_{0}=0\right), \\
c_{n}=\frac{18}{n(n+1)}, & n \geq 1\left(c_{0}=0\right), \\
d_{n}=-\frac{3 n^{2}+3 n-10}{n(n+1)}, & n \geq 1\left(d_{0}=0\right), \\
e_{n}=\frac{n(n+3)}{n+1}, & n \geq 0 .
\end{array}
$$




\section{The integral representations}

As in Part I, we are concerned in this section with the problem of the integral representation of each of the linear functionals $u_{0}, u_{1}$ where $\mathcal{U}={ }^{t}\left(u_{0}, u_{1}\right)$ is the 2-dimensional functional with respect to which the 2-symmetric sequence $\left\{P_{n}\right\}_{n \geq 0}$ defined by (2.30) is 2-orthogonal.

The main result here is

Theorem 4.1. The two linear functionals, $u_{0}$ and $u_{1}$ associated with the 2-classical sequence $\left\{P_{n}\right\}_{n \geq 0}$ defined by (2.30), have the integral representations

$$
\begin{aligned}
& \left\langle u_{0}, f\right\rangle=\int_{-\infty}^{+\infty} U_{0}(x) f(x) d x, \quad f \in \mathcal{P}, \\
& \left\langle u_{1}, f\right\rangle=\int_{-\infty}^{+\infty} U_{1}(x) f(x) d x-\zeta\langle\delta, f\rangle, \quad f \in \mathcal{P},
\end{aligned}
$$

respectively, where the weight functions $U_{0}$ and $U_{1}$ are given by

$$
\begin{gathered}
U_{0}(x)=\frac{3 \sqrt{3}}{4 \pi} Y(x(1-x))\left(1-x^{3}\right)^{-1 / 2}\left[\left(1-\sqrt{1-x^{3}}\right)^{1 / 3}+\left(1+\sqrt{1-x^{3}}\right)^{1 / 3}\right] \\
+3 e^{-x} Y(x)\left[\lambda_{1} \sqrt{x} \cos (\sqrt{3} x)+\lambda_{2} x^{2} \sin (\sqrt{3} x)\right], \\
\begin{aligned}
U_{1}(x)=\frac{9 \sqrt{3}}{8 \pi} Y(x(1-x))\left\{2 x^{2}\left(1-x^{3}\right)^{-1 / 2}\left[\left(1-\sqrt{1-x^{3}}\right)^{1 / 3}+\left(1+\sqrt{1-x^{3}}\right)^{1 / 3}\right]\right. \\
\left.-\left[\left(1-\sqrt{1-x^{3}}\right)^{2 / 3}-\left(1+\sqrt{1-x^{3}}\right)^{2 / 3}\right]\right\} \\
+9 Y(x) e^{-x}\left\{\left[\lambda_{1} x^{-1 / 2}\left(x^{4}-\frac{3}{2} x^{3}-x+\frac{1}{2}\right)-\lambda_{2} \sqrt{3} x^{2}\left(x^{3}-1\right)\right] \cos (\sqrt{3} x)\right. \\
\left.+\left[-\sqrt{3} \lambda_{1} x^{1 / 2}\left(x^{3}-1\right)+\lambda_{2} x\left(x^{4}-3 x^{3}-x+2\right)\right] \sin (\sqrt{3} x)\right\}
\end{aligned}
\end{gathered}
$$

where $Y(x)=\left\{\begin{array}{ll}0 & \text { if } x \leq 0, \\ 1 & \text { if } x>0,\end{array}\right.$ and $\lambda_{1}, \lambda_{2}$, and $\zeta$ are constants which we determine below.

In this section, we apply the method similar to that used in Section 4 of [6].

According to $[4,5]$, the 2 -dimensional functional $\mathcal{U}$ is characterized by the fact that it satisfies a vector distributional equation of the form

$$
\Psi \mathcal{U}+D(\Phi \mathcal{U})=0
$$

where the $2 \times 2$ matrices $\Psi, \Phi$ are defined by (see [4, Section 6$]$ for the explicit formulas)

with

$$
\Psi(x)=\left(\begin{array}{cc}
0 & 1 \\
\frac{2}{3 \gamma} x & \frac{4 \alpha}{3 \gamma}
\end{array}\right), \quad \Phi(x)=\left(\begin{array}{ll}
\phi_{0}^{0}(x) & \phi_{0}^{1}(x) \\
\phi_{1}^{0}(x) & \phi_{1}^{1}(x)
\end{array}\right)
$$

$$
\begin{array}{ll}
\phi_{0}^{0}(x)=\frac{\alpha}{3 \gamma} x+3, & \phi_{0}^{1}(x)=-2 x-\frac{\alpha}{3 \gamma} \\
\phi_{1}^{0}(x)=-\frac{2}{3 \gamma} x^{2}-\frac{\alpha^{2}}{3 \gamma^{2}} x-\frac{\alpha}{\gamma}, & \phi_{1}^{1}(x)=-\frac{7 \alpha}{3 \gamma} x+\frac{2 \alpha^{3}}{3 \gamma^{2}}+3 .
\end{array}
$$


In the rest of this section, we put $\alpha=0$ and $\gamma=4 / 27$. Hence $\left\{P_{n}\right\}_{n \geq 0}$ is reducible to 2 -symmetric 2-OPS, and the above distributional system satisfied by $\mathcal{U}$ becomes

$$
D\left[\left(\begin{array}{cc}
3 & -2 x \\
-\frac{9}{2} x^{2} & 3
\end{array}\right)\left(\begin{array}{l}
u_{0} \\
u_{1}
\end{array}\right)\right]+\left(\begin{array}{cc}
0 & 1 \\
\frac{9}{2} x & 0
\end{array}\right)\left(\begin{array}{l}
u_{0} \\
u_{1}
\end{array}\right)=\left(\begin{array}{l}
0 \\
0
\end{array}\right)
$$

which gives

$$
\begin{aligned}
& 3 u_{0}^{\prime}-2 x u_{1}^{\prime}-u_{1}=0, \\
& 3 x^{2} u_{0}^{\prime}-2 u_{1}^{\prime}+3 x u_{0}=0 .
\end{aligned}
$$

From this, the linear functionals $u_{0}$ and $u_{1}$ satisfy the second-order differential equations

$$
\begin{aligned}
& \left(x^{3}-1\right) u_{0}^{\prime \prime}+\frac{9}{2} x^{2} u_{0}^{\prime}+\frac{5}{2} x u_{0}=0 \\
& x\left(x^{3}-1\right) u_{1}^{\prime \prime}+\left(\frac{7}{2} x^{3}+1\right) u_{1}^{\prime}+x^{2} u_{1}=0
\end{aligned}
$$

respectively, with

$$
u_{1}=-3\left(x^{3}-1\right) u_{0}^{\prime}-3 x^{2} u_{0}
$$

As in Part I, in order to find integral representations of the functionals $u_{0}$ and $u_{1}$, we also will make use of the operator $\sigma_{3}$ and proceed as follows.

(a) We start by determining the weight function $V$ representing the functional $u_{0}^{[1]}=$ $\sigma_{3}\left(u_{0}\right)$, that is,

$$
\left\langle\sigma_{3}\left(u_{0}\right), f\right\rangle=\int_{\mathcal{C}} V(x) f(x) d x, \quad f \in \mathcal{P} .
$$

We show that the function $V$ satisfies a Gaussian hypergeometric equation.

(b) We first give an integral representation of the functional $u_{0}^{[2]}=\sigma_{3}\left(x u_{1}\right)$. We obtain, by means of (4.8), a weight function in terms of $V$ and $V^{\prime}$. Next, by determining the other functionals $u_{j}^{[i]}$ in terms of $u_{0}^{[1]}$ and $u_{0}^{[2]}$, we easily obtain their integral representations.

(c) Finally, we return to the functionals $u_{0}, u_{1}$ and give the proof of Theorem 4.1.

4.1. The determination of the weight function $V$. From the equation (4.6), we can write

$$
D\left[D\left(x^{3}-1\right) u_{0}-\frac{3}{2} x^{2} u_{0}\right]-\frac{1}{2} x u_{0}=0 .
$$

Left-multiplying the last equation by $x^{2}$, it follows that

$$
D\left[D\left(x^{2}\left(x^{3}-1\right) u_{0}\right)-\left(\frac{11}{2} x^{4}-4 x\right) u_{0}\right]+\left(\frac{9}{2} x^{3}-2\right) u_{0}=0 .
$$

From this, by applying $\sigma_{3}$ and using the properties (1.16)-(1.18), we obtain that

$$
D\left[D\left(x^{2}(x-1) \sigma_{3}\left(u_{0}\right)\right)-\left(\frac{5}{2} x^{2}-2 x\right) \sigma_{3}\left(u_{0}\right)\right]+\left(\frac{1}{2} x-\frac{2}{9}\right) \sigma_{3}\left(u_{0}\right)=0 .
$$

Now, to determine an integral representation of the functional $u_{0}^{[1]}=\sigma_{3}\left(u_{0}\right)$, consider

$$
\left\langle u_{0}^{[1]}, f\right\rangle=\int_{\mathcal{C}} V(x) f(x) d x, \quad f \in \mathcal{P} .
$$


The problem is to find the weight function $V$ such that the following two conditions on $V$ hold (see conditions (4.5),(4.6), Part I):

$$
\begin{gathered}
{\left[x^{2}(x-1) V(x) f^{\prime}(x)-\left\{\left[x^{2}(x-1) V(x)\right]^{\prime}-\left(\frac{5}{2} x^{2}-2 x\right) V(x)\right\} f(x)\right]_{\mathcal{C}}=0, \quad f \in \mathcal{P},} \\
x^{2}(x-1) V^{\prime \prime}(x)+x\left(\frac{7}{2} x-2\right) V^{\prime}(x)+\left(\frac{3}{2} x-\frac{2}{9}\right) V(x)=\lambda g(x)
\end{gathered}
$$

where $\lambda$ is an arbitrary constant and $g$ is a function representing the null form, i.e., $\int_{\mathcal{C}} g(x) x^{n} d x=0, n \geq 0$.

In the sequel, we let $\lambda=0$; then (4.13) becomes

$$
x^{2}(x-1) V^{\prime \prime}(x)+x\left(\frac{7}{2} x-2\right) V^{\prime}(x)+\left(\frac{3}{2} x-\frac{2}{9}\right) V(x)=0 .
$$

For solving this equation, we first let $V(x)=x^{\mu} Z(x)$. Hence, by substituting in (4.14), we obtain

$$
\begin{aligned}
& x^{2}(x-1) Z^{\prime \prime}(x)+x\left[\left(2 \mu+\frac{7}{2}\right) x-2 \mu-2\right] Z^{\prime}(x) \\
&+\left[\left(\mu(\mu-1)+\frac{7}{2} \mu+\frac{3}{2}\right) x-\left(\mu+\frac{1}{3}\right)\left(\mu+\frac{2}{3}\right)\right] Z(x)=0 .
\end{aligned}
$$

For $\mu=-1 / 3$, the above becomes

$$
x(1-x) Z^{\prime \prime}(x)+\left(\frac{4}{3}-\frac{17}{6} x\right) Z^{\prime}(x)-\frac{7}{9} Z(x)=0,
$$

which is a hypergeometric equation with general solution given by (see, e.g., [10])

$$
Z(x)=C_{1} Z_{1}(x)+C_{2} Z_{2}(x)
$$

where

$$
\begin{aligned}
& Z_{1}(x)={ }_{2} F_{1}\left(\frac{2}{3} ; \frac{7}{6} ; \frac{4}{3}, x\right)=2^{1 / 3}(1-x)^{-1 / 2}(1+\sqrt{1-x})^{-1 / 3} \\
& Z_{2}(x)=x^{-1 / 3} F_{1}\left(\frac{1}{3} ; \frac{5}{6} ; \frac{2}{3}, x\right)=2^{-1 / 3} x^{-1 / 3}(1-x)^{-1 / 2}(1+\sqrt{1-x})^{1 / 3}
\end{aligned}
$$

and $C_{1}, C_{2}$ are two constants which will be determined below.

Then the general solution of the equation (4.14) may be written as

$$
V(x)=C_{1} \frac{2^{1 / 3} x^{-1 / 3}}{\sqrt{1-x}}(1+\sqrt{1-x})^{-1 / 3}+C_{2} \frac{2^{-1 / 3} x^{-2 / 3}}{\sqrt{1-x}}(1+\sqrt{1-x})^{1 / 3} .
$$

The two constants $C_{1}$ and $C_{2}$, of course, depend on the choice of the path $\mathcal{C}$ and the normalization condition

$$
\left\langle u_{0}^{[1]}, 1\right\rangle=\int_{\mathcal{C}} V(x) d x=1 .
$$

Choose $\mathcal{C}=] 0,1[$, so that the condition (4.12) holds if and only if

$$
C_{1}-2^{-2 / 3} C_{2}=0 \text {. }
$$


Indeed, by differentiating (4.16), we obtain

$$
\begin{aligned}
V^{\prime}(x)=- & \frac{2^{1 / 3}}{3} C_{1} x^{-4 / 3}(1-x)^{-1 / 2}(1+\sqrt{1-x})^{-1 / 3} \\
& -\frac{2^{2 / 3}}{3} C_{2} x^{-5 / 3}(1-x)^{-1 / 2}(1+\sqrt{1-x})^{1 / 3} \\
& +2^{-2 / 3} C_{1} x^{-1 / 3}(1-x)^{-3 / 2}(1+\sqrt{1-x})^{-1 / 3} \\
& +2^{-4 / 3} C_{2} x^{-2 / 3}(1-x)^{-3 / 2}(1+\sqrt{1-x})^{1 / 3} \\
& +\frac{2^{-2 / 3}}{3} C_{1} x^{-1 / 3}(1-x)^{-1}(1+\sqrt{1-x})^{-4 / 3} \\
& -\frac{2^{-4 / 3}}{3} C_{2} x^{-2 / 3}(1-x)^{-1}(1+\sqrt{1-x})^{-2 / 3}
\end{aligned}
$$

Now, it is easy to verify that

$$
\begin{aligned}
& x^{2}(x-1) V(x) \rightarrow 0 \quad \text { when } x \rightarrow 1^{-} \text {and } x \rightarrow 0^{+}, \\
& \frac{1}{2} x^{2} V(x)-x^{2}(x-1) V^{\prime}(x) \rightarrow 0 \quad \text { when } x \rightarrow 0^{+},
\end{aligned}
$$

and

$$
\frac{1}{2} x^{2} V(x)-x^{2}(x-1) V^{\prime}(x) \rightarrow \frac{1}{3}\left\{-2^{-2 / 3} C_{1}+2^{-4 / 3} C_{2}\right\} \quad \text { when } x \rightarrow 1^{-} .
$$

Then the condition (4.12) holds if and only if (4.17) holds.

Consequently (4.16) becomes

$$
\begin{aligned}
V(x) & =2^{1 / 3} C_{1}(1-x)^{-1 / 2}\left\{x^{-1 / 3}(1+\sqrt{1-x})^{-1 / 3}+x^{-2 / 3}(1+\sqrt{1-x})^{1 / 3}\right\} \\
& =2^{1 / 3} C_{1} x^{-2 / 3}(1-x)^{-1 / 2}\left\{(1-\sqrt{1-x})^{1 / 3}+(1+\sqrt{1-x})^{1 / 3}\right\}, \quad 0<x<1 .
\end{aligned}
$$

Now, we determine the constant $C_{1}$ by using the normalization condition $\int_{0}^{1} V(x) d x=1$.

Indeed, from (4.18) we have

or

$$
2^{1 / 3} C_{1}\left\{\int_{0}^{1} \frac{x^{-2 / 3}}{\sqrt{1-x}}(1-\sqrt{1-x})^{1 / 3} d x+\int_{0}^{1} \frac{x^{-2 / 3}}{\sqrt{1-x}}(1+\sqrt{1-x})^{1 / 3} d x\right\}=1
$$

$$
2^{1 / 3} C_{1}\left\{J_{1}+J_{2}\right\}=1
$$

where

$$
J_{1}=\int_{0}^{1} \frac{x^{-2 / 3}}{\sqrt{1-x}}(1-\sqrt{1-x})^{1 / 3} d x \quad \text { and } \quad J_{2}=\int_{0}^{1} \frac{x^{-2 / 3}}{\sqrt{1-x}}(1+\sqrt{1-x})^{1 / 3} d x .
$$

Using the transformation $t=\sqrt{1-x}$, the two integrals $J_{1}$ and $J_{2}$ can be written as

and

$$
J_{1}=2 \int_{0}^{1}(1+t)^{-2 / 3}(1-t)^{-1 / 3} d t
$$

$$
J_{2}=2 \int_{0}^{1}(1+t)^{-1 / 3}(1-t)^{-2 / 3} d t=2 \int_{-1}^{0}(1+t)^{-2 / 3}(1-t)^{-1 / 3} d t
$$


Then

$$
J_{1}+J_{2}=2 \int_{-1}^{1}(1+t)^{-2 / 3}(1-t)^{-1 / 3} d t=\frac{4 \pi}{\sqrt{3}} .
$$

It follows that $C_{1}=\frac{2^{-1 / 3} \sqrt{3}}{4 \pi}$. By substituting this in (4.18), we obtain

$$
V(x)=\frac{\sqrt{3}}{4 \pi} x^{-2 / 3}(1-x)^{-1 / 2}\left\{(1-\sqrt{1-x})^{1 / 3}+(1+\sqrt{1-x})^{1 / 3}\right\}, \quad 0<x<1 .
$$

It is clear that $V>0$ on $] 0,1\left[\right.$, and then the linear functional $u_{0}^{[1]}$ is positive definite.

4.2. The representations of the functionals $u_{0}^{[i]}, i=2,3$, and $u_{1}^{[i]}, i=1,2,3$. We now are looking for an integral representation of each of the linear functionals $u_{0}^{[i]}$, $i=2,3$ and $u_{1}^{[i]}, i=1,2,3$, where $\mathcal{U}^{[1]}={ }^{t}\left(u_{0}^{[1]}, u_{1}^{[1]}\right), \mathcal{U}^{[2]}={ }^{t}\left(u_{0}^{[2]}, u_{1}^{[2]}\right)$, and $\mathcal{U}^{[3]}=$ ${ }^{t}\left(u_{0}^{[3]}, u_{1}^{[3]}\right)$ are the 2-dimensional functionals with respect to which the components $\left\{P_{n}^{[1]}\right\}_{n \geq 0},\left\{P_{n}^{[2]}\right\}_{n \geq 0}$, and $\left\{P_{n}^{[3]}\right\}_{n \geq 0}$, are 2-orthogonal, respectively.

From (2.18), when $d=2$, we have

$$
u_{j}^{[i]}=\sigma_{3}\left(x^{i-1} u_{3 j+i-1}\right), \quad j=0,1, i=1,2,3 .
$$

This leads to

$$
\left(\begin{array}{c}
u_{0}^{[1]} \\
u_{1}^{[1]}
\end{array}\right)=\left(\begin{array}{l}
\sigma_{3}\left(u_{0}\right) \\
\sigma_{3}\left(u_{3}\right)
\end{array}\right), \quad\left(\begin{array}{l}
u_{0}^{[2]} \\
u_{1}^{[2]}
\end{array}\right)=\left(\begin{array}{l}
\sigma_{3}\left(x u_{1}\right) \\
\sigma_{3}\left(x u_{4}\right)
\end{array}\right), \quad \text { and } \quad\left(\begin{array}{c}
u_{0}^{[3]} \\
u_{1}^{[3]}
\end{array}\right)=\left(\begin{array}{l}
\sigma_{3}\left(x^{2} u_{2}\right) \\
\sigma_{3}\left(x^{2} u_{5}\right)
\end{array}\right) .
$$

The representation of the linear functional $u_{0}^{[1]}$ is given in the above subsection. To find an integral representation of each of the functionals $u_{j}^{[i]}$, we proceed as follows.

- We give explicit expressions of the functionals $u_{j}^{[i]}$ in terms of $u_{0}^{[1]}$ and $u_{0}^{[2]}$.

- We determine a weight function representing the linear functional $u_{0}^{[2]}$ in terms of $V$ and $V^{\prime}$.

- With the aid of the representations of $u_{0}^{[1]}$ and $u_{0}^{[2]}$, we obtain the integral representations of the other functionals.

Indeed, by virtue of the relation (1.8), we have, for $d=2$,

$$
x u_{n}=u_{n-1}+\beta_{n} u_{n}+\gamma_{n+1}^{1} u_{n+1}+\gamma_{n+1}^{0} u_{n+2}, \quad n \geq 0, \quad\left(u_{-1}=0\right) .
$$

In this case, $\beta_{n}=0, \gamma_{n+1}^{1}=\alpha=0, n \geq 0$, and $\gamma_{1}^{0}=3 \gamma, \gamma_{n+1}^{0}=\gamma(=4 / 27), n \geq 1$. Whence, the above becomes

$$
\begin{aligned}
& \gamma u_{n+2}=x u_{n}-u_{n-1}, \quad n \geq 1, \\
& 3 \gamma u_{2}=x u_{0} .
\end{aligned}
$$

From this, we can successively express $u_{n}$, for any integer $n \geq 3$, in terms of $u_{0}$ and $u_{1}$ :

- for $n=0: u_{2}=3^{-1} \gamma^{-1} x u_{0}$,

- for $n=1: u_{3}=\gamma^{-1}\left(x u_{1}-u_{0}\right)$,

- for $n=2: u_{4}=\gamma^{-1}\left(x u_{2}-u_{1}\right)=-3^{-1} \gamma^{-2}\left(3 \gamma u_{1}-x^{2} u_{0}\right)$,

- for $n=3: u_{5}=\gamma^{-1}\left(x u_{3}-u_{2}\right)=3^{-1} \gamma^{-2}\left(3 x^{2} u_{1}-4 x u_{0}\right)$. 
Therefore, by applying the operator $\sigma_{3}$ and taking into account of (4.8), we obtain, respectively,

$$
\begin{aligned}
& u_{0}^{[3]}=\sigma_{3}\left(x^{2} u_{2}\right)=3^{-1} \gamma^{-1} x u_{0}^{[1]}, \\
& u_{1}^{[1]}=\sigma_{3}\left(u_{3}\right)=\gamma^{-1}\left\{u_{0}^{[2]}-u_{0}^{[1]}\right\}, \\
& u_{1}^{[2]}=\sigma_{3}\left(x u_{4}\right)=-3^{-1} \gamma^{-2}\left\{3 \gamma u_{0}^{[2]}-x u_{0}^{[1]}\right\}, \\
& u_{1}^{[3]}=\sigma_{3}\left(x^{2} u_{5}\right)=3^{-1} \gamma^{-2}\left\{3 x u_{0}^{[2]}-4 x u_{0}^{[1]}\right\} .
\end{aligned}
$$

First, according to the relation (4.8), we easily obtain

$$
u_{0}^{[2]}=\sigma_{3}\left(x u_{1}\right)=-9\left(x(x-1) u_{0}^{[1]}\right)^{\prime}+3(3 x-1) u_{0}^{[1]} .
$$

To determine the integral representation of the functional $u_{0}^{[2]}$, we first need

$$
\left\langle\left(x(x-1) u_{0}^{[1]}\right)^{\prime}, f(x)\right\rangle=-\left\langle x(x-1) u_{0}^{[1]}, f^{\prime}(x)\right\rangle=-\int_{0}^{1} x(x-1) V(x) f^{\prime}(x) d x .
$$

Integration by parts gives

$$
\left\langle\left(x(x-1) u_{0}^{[1]}\right)^{\prime}, f(x)\right\rangle=[x(1-x) V(x) f(x)]_{0}^{1}+\int_{0}^{1}(x(x-1) V(x))^{\prime} f(x) d x .
$$

Since $[x(1-x) V(x) f(x)]_{0}^{1}=0$, we get

$$
\left\langle\left(x(x-1) u_{0}^{[1]}\right)^{\prime}, f(x)\right\rangle=\int_{0}^{1}(x(x-1) V(x))^{\prime} f(x) d x .
$$

Now, from (4.24), we have

$$
\begin{aligned}
\left\langle u_{0}^{[2]}, f\right\rangle & =-9\left\langle\left(x(x-1) u_{0}^{[1]}\right)^{\prime}, f\right\rangle+3\left\langle(3 x-1) u_{0}^{[1]}, f\right\rangle \\
& =-9 \int_{0}^{1}(x(x-1) V(x))^{\prime} f(x) d x+3 \int_{0}^{1}(3 x-1) V(x) f(x) d x \\
& =\int_{0}^{1} U_{0}^{[2]}(x) f(x) d x, \quad f \in \mathcal{P}
\end{aligned}
$$

where

$$
U_{0}^{[2]}(x)=-3(3 x-2) V(x)-9 x(x-1) V^{\prime}(x) .
$$

Or, differentiating (4.19) and substituting into the last equation, we obtain

$$
U_{0}^{[2]}(x)=\frac{3}{2}\left\{x+x^{1 / 3} \sqrt{1-x}\left[(1+\sqrt{1-x})^{1 / 3}-(1-\sqrt{1-x})^{1 / 3}\right]\right\} V(x) .
$$

Thus, it is simple to see that $U_{0}^{[2]}(x)>0$ for $\left.x \in\right] 0,1\left[\right.$. Then the linear functional $u_{0}^{[2]}$ is positive definite.

Finally, by means of (4.20)-(4.23) and using (4.25), one easily finds from (4.21)

$$
U_{1}^{[1]}(x)=-2^{-2} 3^{3}\left\{(9 x-5) V(x)+9 x(x-1) V^{\prime}(x)\right\} .
$$

Remember that $U_{0}^{[1]}=V$, hence we obtain the pair of the weight functions $U_{0}^{[1]}$ and $U_{1}^{[1]}$ representing, respectively, the two functionals $u_{0}^{[1]}$ and $u_{1}^{[1]}$.

From (4.22), we obtain

$$
U_{1}^{[2]}(x)=2^{-4} 3^{4}\left\{(15 x-8) V(x)+12 x(x-1) V^{\prime}(x)\right\} .
$$


This equation with (4.25) gives the pair of weight functions for the two functionals $u_{1}^{[2]}$ and $u_{0}^{[2]}$, respectively.

From (4.20) and (4.23), we obtain

$$
\begin{aligned}
& U_{0}^{[3]}(x)=2^{-2} 3^{2} x V(x) \\
& U_{1}^{[3]}(x)=-2^{-4} 3^{5}\left\{x(27 x-14) V(x)+27 x^{2}(x-1) V^{\prime}(x)\right\} .
\end{aligned}
$$

Since $V(x)>0$ for $x \in] 0,1\left[\right.$, then $U_{0}^{[3]}(x)>0$, so that the linear functional $u_{0}^{[3]}$ is also positive definite.

Again, as in Part I, we observe that the three linear functionals $u_{0}^{[1]}, u_{0}^{[2]}$, and $u_{0}^{[3]}$ are also positive definite. According to this result and those found in $[6,7]$, we can formulate the following conjecture in the case of a 2 -symmetric "classical" sequence:

If the linear functional $u_{0}^{[1]}$ is positive definite, then the linear functionals $u_{0}^{[2]}$ and $u_{0}^{[3]}$ are also positive definite.

4.3. The integral representation of $u_{0}$ and $u_{1}$. Proof of Theorem 4.1. Finally, we return to the initial problem for determining an integral representation of the functional $u_{0}$, and, by virtue of (4.8), we deduce an integral representation of the functional $u_{1}$. First consider

$$
\left\langle u_{0}, f\right\rangle=\int_{-\infty}^{\infty} U_{0}(x) f(x) d x, \quad f \in \mathcal{P} .
$$

We have

$$
\left\langle\sigma_{3}\left(u_{0}\right), f\right\rangle=\int_{-\infty}^{\infty} Y(x(1-x)) V(x) f(x) d x, \quad f \in \mathcal{P} .
$$

In particular, since $\left\langle u_{0}, \sigma_{3} f\right\rangle=\left\langle\sigma_{3}\left(u_{0}\right), f\right\rangle$, we have

$$
\int_{-\infty}^{\infty} U_{0}(x) f\left(x^{3}\right) d x=\int_{-\infty}^{\infty} Y(x(1-x)) V(x) f(x) d x, \quad f \in \mathcal{P},
$$

which also can be written

$$
\int_{-\infty}^{\infty}\left\{Y(x(1-x)) V(x)-\frac{1}{3} U_{0}\left(x^{1 / 3}\right) x^{-2 / 3}\right\} f(x) d x=0, \quad f \in \mathcal{P} .
$$

Hence $Y(x(1-x)) V(x)-U_{0}\left(x^{1 / 3}\right) / 3 x^{2 / 3}$ is a function representing the null functional. Then

$$
Y(x(1-x)) V(x)-\frac{1}{3} x^{-2 / 3} U_{0}\left(x^{1 / 3}\right)=\lambda_{1} g_{1}(x)+\lambda_{2} g_{2}(x)
$$

where $g_{1}$ and $g_{2}$ are two functions which represent the null functional, that is,

$$
\int_{-\infty}^{\infty} x^{n} g_{1}(x) d x=0 \text { and } \int_{-\infty}^{\infty} x^{n} g_{2}(x) d x=0, \quad n \geq 0 .
$$

For example, we can take the two functions $g_{1}$ and $g_{2}$ as follows:

$$
g_{1}(x)=Y(x) x^{-1 / 2} e^{-x^{1 / 3}} \cos \left(\sqrt{3} x^{1 / 3}\right) \quad \text { and } \quad g_{2}(x)=Y(x) e^{-x^{1 / 3}} \sin \left(\sqrt{3} x^{1 / 3}\right) .
$$

Now, by changing $x$ to $x^{3}$ in (4.26), we find

$$
U_{0}(x)=3 x^{2}\left\{Y(x(1-x)) V\left(x^{3}\right)-\lambda_{1} g_{1}\left(x^{3}\right)-\lambda_{2} g_{2}\left(x^{3}\right)\right\} .
$$


Because the functional $u_{0}$ is 2 -symmetric, we necessarily have $\left(u_{0}\right)_{0}=1,\left(u_{0}\right)_{1}=$ $\left(u_{0}\right)_{2}=0$, i.e.,

$$
\left(u_{0}\right)_{\nu}=\int_{-\infty}^{\infty} x^{\nu} U_{0}(x) d x= \begin{cases}1, & \nu=0 \\ 0, & \nu=1,2\end{cases}
$$

The first condition is fulfilled for any values of the constants $\lambda_{1}, \lambda_{2}$. The other two conditions, obtained for $\nu=1$ and $\nu=2$, allow us to determine the constants $\lambda_{1}, \lambda_{2}$, from the system

$$
\begin{aligned}
& \lambda_{1} I_{11}+\lambda_{2} I_{12}=-K_{1}, \\
& \lambda_{1} I_{21}+\lambda_{2} I_{22}=-K_{2}
\end{aligned}
$$

where

$$
I_{\nu \mu}=\int_{0}^{\infty} x^{\nu+2} g_{\mu}\left(x^{3}\right) d x, \quad \nu, \mu=1,2, \quad \text { and } \quad K_{\nu}=\int_{0}^{1} x^{\nu+2} V\left(x^{3}\right) d x, \quad \nu=1,2 .
$$

First, using (4.19) and the transformation $t=\sqrt{1-x}$, we easily can calculate $K_{1}$ and $K_{2}$

$$
K_{1}=\frac{1}{3} \int_{0}^{1} x^{1 / 3} V(x) d x=\frac{\sqrt{3}}{6 \pi} \int_{0}^{1}\left(1-t^{2}\right)^{-1 / 3}\left[(1+t)^{1 / 3}+(1-t)^{1 / 3}\right] d t=\frac{2^{2 / 3} \sqrt{3}}{4 \pi}
$$

and

$$
K_{2}=\frac{1}{3} \int_{0}^{1} x^{2 / 3} V(x) d x=\frac{\sqrt{3}}{12 \pi} \int_{0}^{1}\left(1-t^{2}\right)^{-1 / 3}\left[(1+t)^{1 / 3}+(1-t)^{1 / 3}\right] d t=\frac{2^{1 / 3} \sqrt{3}}{4 \pi} .
$$

Secondly, according to the two formulas [8]

$$
\begin{aligned}
\int_{0}^{\infty} x^{p-1} e^{-a x} \cos (m x) d x & =\frac{\Gamma(p) \cos (p \theta)}{\left(a^{2}+m^{2}\right)^{p / 2}}, \\
\int_{0}^{\infty} x^{p-1} e^{-a x} \sin (m x) d x & =\frac{\Gamma(p) \sin (p \theta)}{\left(a^{2}+m^{2}\right)^{p / 2}}
\end{aligned}
$$

where $p, a, m>0, \sin \theta=m / r, \cos \theta=a / r, 0<\theta<\pi / 2, r=\left(a^{2}+m^{2}\right)^{1 / 2}$, we obtain that

$$
\begin{array}{ll}
I_{11}=-\sqrt{2 \pi} 2^{-6} 3^{3 / 2}, & I_{12}=-2^{-4} 3^{3 / 2}, \\
I_{21}=-5 \sqrt{2 \pi} 2^{-8} 3^{3 / 2}, & I_{22}=-2^{-3} 3^{3 / 2} .
\end{array}
$$

Thus, the system (4.28) gives $\Delta \lambda_{1}=K_{2} I_{12}-K_{1} I_{22}$ and $\Delta \lambda_{2}=K_{1} I_{21}-K_{2} I_{11}$ where $\Delta=I_{11} I_{22}-I_{21} I_{12}=3^{4} 4^{-6} \sqrt{2 \pi}$.

It follows that

$$
\lambda_{1}=\frac{1}{\pi} \sqrt{\frac{2}{\pi}} \frac{2^{6}}{3^{2}}\left\{2^{2 / 3}-2^{-2 / 3}\right\} \quad \text { and } \quad \lambda_{2}=\frac{1}{\pi} \frac{2^{4}}{3^{2}}\left\{5 \times 2^{1 / 3}-2^{-1 / 3}\right\} .
$$

Whence, from (4.27), we obtain that

$$
\begin{aligned}
U_{0}(x)= & \frac{3 \sqrt{3}}{4 \pi} Y(x(1-x)) \frac{\left(1-\sqrt{1-x^{3}}\right)^{1 / 3}+\left(1+\sqrt{1-x^{3}}\right)^{1 / 3}}{\sqrt{1-x^{3}}} \\
& +3 e^{-x} Y(x)\left[\lambda_{1} \sqrt{x} \cos (\sqrt{3} x)+\lambda_{2} x^{2} \sin (\sqrt{3} x)\right] .
\end{aligned}
$$


We now look for the representation of the functional $u_{1}$. At first, from (4.8), we have

$$
u_{1}=-3\left(x^{3}-1\right) u_{0}^{\prime}-3 x^{2} u_{0} .
$$

Then, using (4.27), we can write

$$
\begin{aligned}
\left\langle u_{1}, f\right\rangle & =3\left\langle u_{0},\left(\left(x^{3}-1\right) f(x)\right)^{\prime}\right\rangle-3\left\langle u_{0}, x^{2} f(x)\right\rangle \\
& =3 \int_{-\infty}^{\infty} U_{0}(x)\left[\left(x^{3}-1\right) f(x)\right]^{\prime} d x-3 \int_{-\infty}^{\infty} U_{0}(x) x^{2} f(x) d x \\
& =I_{1}+I_{2}+I_{3}
\end{aligned}
$$

with

$$
\begin{aligned}
& I_{1}=9 \int_{0}^{1} x^{2} V\left(x^{3}\right)\left[\left(x^{3}-1\right) f(x)\right]^{\prime} d x \\
& I_{2}=9 \int_{0}^{\infty} x^{2}\left[\lambda_{1} g_{1}\left(x^{3}\right)+\lambda_{2} g_{2}\left(x^{3}\right)\right]\left[\left(x^{3}-1\right) f(x)\right]^{\prime} d x \\
& I_{3}=-3 \int_{-\infty}^{\infty} x^{2} U_{0}(x) f(x) d x
\end{aligned}
$$

Hence, by integration by parts

$$
I_{1}=\left[9 x^{2}\left(x^{3}-1\right) V\left(x^{3}\right) f(x)\right]_{0}^{1}-9 \int_{0}^{1}\left(x^{3}-1\right)\left[x^{2} V\left(x^{3}\right)\right]^{\prime} f(x) d x .
$$

Now, from (4.19), we have

$$
x^{2} V\left(x^{3}\right)=\frac{\sqrt{3}}{4 \pi}\left(1-x^{3}\right)^{-1 / 2}\left[\left(1-\sqrt{1-x^{3}}\right)^{1 / 3}+\left(1+\sqrt{1-x^{3}}\right)^{1 / 3}\right] .
$$

Then $\left[9 x^{2} V\left(x^{3}\right)\left(1-x^{3}\right) f(x)\right]_{0}^{1}=\frac{2^{1 / 3} 3^{5 / 2}}{4 \pi} f(0)$. Now, by differentiating (4.30) and using (4.19), we obtain

$$
\begin{aligned}
I_{1}= & \frac{2^{\frac{1}{3}} 3^{\frac{5}{2}}}{4 \pi} f(0)+\frac{27 \sqrt{3}}{8 \pi} \int_{0}^{1}\left\{x^{2}\left(1-x^{3}\right)^{-\frac{1}{2}}\left[\left(1-\sqrt{1-x^{3}}\right)^{\frac{1}{3}}+\left(1+\sqrt{1-x^{3}}\right)^{\frac{1}{3}}\right]\right. \\
& \left.-\frac{1}{3}\left[\left(1-\sqrt{1-x^{3}}\right)^{2 / 3}-\left(1+\sqrt{1-x^{3}}\right)^{2 / 3}\right]\right\} f(x) d x .
\end{aligned}
$$

Next, it is simple to see that

$$
\begin{aligned}
I_{2}= & 9 \int_{0}^{\infty} e^{-x}\left\{\left[\lambda_{1} x^{-1 / 2}\left(x^{4}-\frac{1}{2} x^{3}-x+\frac{1}{2}\right)-\lambda_{2} \sqrt{3} x^{2}\left(x^{3}-1\right)\right] \cos (\sqrt{3} x)\right. \\
& \left.+\left[-\sqrt{3} \lambda_{1} x^{1 / 2}\left(x^{3}-1\right)+\lambda_{2} x\left(x^{4}-2 x^{3}-x+2\right)\right] \sin (\sqrt{3} x)\right\} f(x) d x
\end{aligned}
$$

and

$$
\begin{aligned}
I_{3}=- & \frac{9 \sqrt{3}}{4 \pi} \int_{0}^{1} x^{2}\left(1-x^{3}\right)^{-1 / 2}\left[\left(1-\sqrt{1-x^{3}}\right)^{1 / 3}+\left(1+\sqrt{1-x^{3}}\right)^{1 / 3}\right] f(x) d x \\
& -9 \int_{0}^{\infty} x^{2} e^{-x}\left[\lambda_{1} x^{1 / 2} \cos (\sqrt{3} x)+\lambda_{2} x^{2} \sin (\sqrt{3} x)\right] f(x) d x
\end{aligned}
$$


Whence, from (4.29), we easily obtain

$$
\left\langle u_{1}, f\right\rangle=\int_{-\infty}^{\infty} U_{1}(x) f(x) d x-\zeta\langle\delta, f\rangle
$$

where $\zeta=2^{-5 / 3} 3^{5 / 2} \pi^{-1}$, and the weight function $U_{1}$ is given by (4.4).

\section{References}

1. W. A. Al-Salam, Characterization theorems for orthogonal polynomials. In: Orthogonal Polynomials: Theory and Practice (Ed. P. Nevai) C294, pp.1-23, Kluwer Academic, 1990.

2. T. S. Chihara, An Introduction to Orthogonal Polynomials, Gordon and Breach, New York, 1978.

3. K. Douak, The relation of the d-orthogonal polynomials to the Appell polynomials, J. Comput. Appl. Math. 70 (1996), 279-295.

4. K. Douak and P. Maroni, Les polynômes orthogonaux "classiques" de dimension deux, Analysis 12 (1992), 71-107.

5. _ Une caractérisation des polynômes d-orthogonaux "classiques", J. Approx. Theory 82 (1995), 177-204.

6. _ On d-orthogonal Tchebychev polynomials, I, Appl. Num. Math. 24 (1997), 23-53.

7. _ Integral representations of the two linear functionals associated with 2-orthogonal Hermite polynomials, (in preparation).

8. H. B. Dwight, Tables of Integral and Other Mathematical Data, 4th ed., Macmillan, New-York, 1961.

9. G. Faber, Über polynomische Entwicklungen, Math. Ann. 57 (1903), 389-408.

10. N. N. Lebedev, Special Functions and Their Applications, Prentice-Hall, N.J., 1965.

11. P. Maroni, L'orthogonalité et les récurrences de polynômes d'ordre supérieur à deux, Ann. Fac. Sci. Toulouse 10 (1989), 105-139.

12. _ Two-dimensional orthogonal polynomials, their associated sets and the co-recursive sets, Numer. Algorithms 3 (1992), 299-312.

13. J. Van Iseghem, Approximants de Padé vectoriels, Thèse d'état, Univ. des Sciences et techniques de Lille-Flandre-Artois, 1987.

Université Pierre et Marie CURIE - C.N.R.S., Laboratoire D'ANalyse NumÉrique, TOUR 55-65 -5Ème Étage, 4 Place Jussieu, 75252 PARIS Cedex 05, FRANCE 